\title{
CAMPOS DE ALTITUDE NA SERRA DO MAR PARANAENSE: ASPECTOS FLORÍSTICOS
}

\author{
Alan Yukio Mocochinski ${ }^{1}$, Maurício Bergamini Scheer ${ }^{2}$ \\ ${ }^{1}$ Eng. Florestal, M.Sc., Curitiba, PR, Brasil - aymocochinski@gmail.com \\ ${ }^{2}$ Eng. Florestal, M.Sc., Doutorando em Engenharia Florestal, UFPR, SANEPAR, Curitiba, PR, Brasil - mauriciobs@sanepar.com.br
}

Recebido para publicação: 15/08/2007 - Aceito para publicação: 01/04/2008

\begin{abstract}
Resumo
O presente trabalho teve o objetivo de estudar a composição florística de seis áreas de ocorrência de campos de altitude na Serra do Mar no estado do Paraná. Foram detectadas 280 espécies vegetais vasculares, pertencentes a 73 famílias, sendo 63 angiospermas (260 espécies), 8 pteridófitas (18 espécies) e 2 gimnospermas ( 2 espécies). A família com maior riqueza específica foi Asteraceae, com 44 espécies $(15,7 \%$ do total), seguida por Poaceae $(31 ; 11 \%)$, Melastomataceae $(17 ; 6 \%)$ e Cyperaceae $(16 ; 5,7 \%)$. A Serra do Ibitiraquire, onde ocorre o maior trecho da formação estudada, apresentou a maior riqueza de espécies (224 espécies), e o menor trecho, na Serra da Pedra Branca do Araraquara, apresentou a menor riqueza (71). Apenas 17 espécies (6\% do total) foram detectadas em todas as áreas e 137 espécies (49\%) são exclusivas de uma das áreas. Observou-se uma associação negativa e moderada entre a similaridade florística e a distância entre as áreas de ocorrência. Também foram reportadas algumas ameaças aos Campos de Altitude no Paraná, com destaque para a invasão biológica por Pinus sp., a conduta inadequada em ambientes de montanha e as obras de infra-estrutura de telecomunicações.

Palavras-chave: Vegetação altomontana; florística; campos de altitude; Serra do Mar; similaridade florística.
\end{abstract}

\begin{abstract}
High-altitude grasslands (campos de altitude) on the Serra do MarMountain in Southern Brazil: floristic aspects. The floristic of high-altitude grasslands (campos de altitude) of the "Serra do Mar" in the state of Parana was studied. In a total of six investigated areas, 280 vascular species were detected. They belong to 73 botanic families, in which 63 are angiosperms (260 species), 8 are pteridophytes (18 species) and 2 are gymnosperms. Asteraceae was the richest family, with 44 species $(15.7 \%$ of the total), followed by Poaceae $(31 ; 11 \%)$, Melastomataceae $(17 ; 6 \%)$ and Cyperaceae $(16$; $5.7 \%)$. The Serra do Ibitiraquire presents the largest area of "campos de altitude" and the highest richness of species (224 species). Serra da Pedra Branca do Araraquara presents the lowest number of species (71 species) probably due to the smallest grassland area. Only 17 species $(6 \%)$ were found on the six sites and 137 species (49\%) were detected on just one site. A moderate negative correlation was observed between floristic similarity and the geographic distance among the high-altitude grassland areas. The biological contamination (mostly by Pinus sp.), the human inadequate behavior in mountain environments and telecommunication facilities are the most important threats to the highaltitude grassland in Paraná.

Keywords: High-altitude grasslands; Serra do Mar Mountain; high mountain environments; "Brazilian Páramos".
\end{abstract}

\section{INTRODUÇ̃̃O}

O sistema de montanhas da Serra do Mar constitui a mais destacada feição orográfica da borda atlântica do continente sul-americano (ALMEIDA; CARNEIRO, 1998). Em sua porção no estado do Paraná, é coberta predominantemente pela Floresta Ombrófila Densa Atlântica (IBGE, 1992; RODERJAN et al., 2002). Acima do limite de ocorrência das florestas, e por vezes entremeando-as, ocorre uma formação vegetacional de fisionomia campestre e subarbustiva: os campos de altitude. Várias denominações já foram empregadas para descrever essa formação, podendo-se citar campos alpinos 
(BARRETO, citado por CAIAFA; SILVA, 2005), refúgios vegetacionais altomontanos (VELOSO, 1991; IBGE, 1992), refúgio ecológico (LEITE, 1994) e campos altimontanos (RIZZINI, 1979), entre outras designações. Neste trabalho, adotou-se o termo campo de altitude, tal como proposto por Ferri (1980) e difundido entre diversos autores (MARTINELLI, 1996; SAFFORD, 1999a, 1999b; OLIVEIRA-FILHO, 1999; TRAMUJAS, 2000; CAIAFA; SILVA, 2005).

Os campos de altitude estão inseridos na complexidade do Bioma Mata Atlântica e ocorrem nas porções mais elevadas da Serra do Mar, nos estados de Santa Catarina, Paraná, São Paulo e Rio de Janeiro, e da Serra da Mantiqueira, com área total de distribuição estimada em $350 \mathrm{~km}^{2}$ (SAFFORD, 1999a). Sua ocorrência junto das formações florestais circundantes deve-se a razões em grande parte relacionadas ao clima e apenas secundariamente às condições pedológicas (RIZZINI, citado por RIBEIRO; MEDINA, 2002). As causas de sua formação são complexas e remetem a uma paisagem campestre antiga, do final do Pleistoceno, quando o clima frio e seco dominava a região sudeste do continente sul-americano (SAFFORD, 1999b). Conforme observou Safford (1999a), o elevado número de espécies endêmicas nessa formação indica que esses ambientes são relativamente antigos e não apenas produtos de distúrbios antrópicos recentes. Nesse mesmo sentido, evidências paleobotânicas sugerem que os campos de altitude têm coberto os cumes do Sudeste do Brasil ininterruptamente desde o final do Pleistoceno (BEHLING, citado por SAFFORD, 1999a). Sua estrutura e composição florística são semelhantes à da estepe gramíneo-lenhosa e à do componente herbáceo da savana (TRAMUJAS, 2000). Os campos de altitude também guardam semelhanças fisionômicas e ecológicas com os campos rupestres, mas diferem pela composição florística e quanto à litologia predominante (CAIAFA; SILVA, 2005). Os campos rupestres ocorrem predominantemente sobre rochas quartzíticas e areníticas, enquanto os campos de altitude ocorrem predominantemente sobre rochas graníticas e migmatíticas.

Os campos de altitude desenvolvem-se em condições ambientais peculiares, decorrentes dos efeitos da altitude e do relevo acidentado. A altitude elevada acarreta temperaturas mais baixas e ventos mais velozes. Paralelamente, o relevo acidentado origina solos rasos e forma um mosaico de micro-hábitats. Essas condições, distintas do contexto do entorno, podem ocasionar níveis elevados de endemismo. Martinelli (1996), estudando seis trechos de ocorrência dos campos de altitude nas Serras do Mar e da Mantiqueira no Sudeste do Brasil, detectou taxas de endemismos variando entre 12 e 22\% em cada área. Em uma análise de endemismos restritos, ou seja, espécies ocorrentes apenas na área estudada, o mesmo autor encontrou taxas entre 2 e $11 \%$, sendo este último valor medido na região do planalto do Itatiaia.

Pela dificuldade de acesso e adversidade das condições ambientais, os campos de altitude permaneceram, em grande parte, a salvo dos processos de conversão dos ambientes naturais para usos antrópicos. Dessa forma, consistem em uma das raras amostras da natureza bem conservada nas regiões Sul e Sudeste do Brasil. Entretanto, sua integridade vem sendo ameaçada por processos como a contaminação biológica e a pressão do turismo. Ao considerar os elevados níveis de endemismo, a singularidade de seus processos ecológicos e sua ocorrência restrita, distribuindo-se no Paraná por algumas dezenas de quilômetros quadrados, fica clara a importância de ações para a conservação desse tipo vegetacional. Para tanto, conhecimentos básicos, que hoje são escassos, são fundamentais. Até o presente, os esforços de coleta foram mais intensos em áreas de fácil acesso e os levantamentos publicados referem-se a amostragens pontuais.

Reconhecendo essa lacuna no conhecimento da diversidade biológica paranaense e brasileira, o presente trabalho teve o objetivo de realizar o levantamento da composição florística de seis áreas de ocorrência dos campos de altitude na Serra do Mar paranaense. Com os resultados, espera-se contribuir para a ampliação dos conhecimentos sobre esse ecossistema e fortalecer as ações para sua conservação.

\section{MATERIAL E MÉTODOS}

\section{Áreas de estudo}

Serra do Mar no Paraná

A Serra do Mar no Paraná configura uma cadeia de montanhas com cimos elevados acima de 1800 m de altitude (ALMEIDA; CARNEIRO, 1998; AB'SÁBER, 2000). Maack (2002) a descreve como uma serra marginal típica que separa o planalto da planície litorânea. Ela se eleva de 500 a 1000 metros sobre o nível médio do planalto, que se encontra a cerca de 900 metros sobre o nível do mar (snm). No Paraná, ela é caracterizada por vários conjuntos de montanhas, que constituem subserras, denominadas 
regionalmente de serras (por exemplo, Serra do Marumbi e Serra do Ibitiraquire). A litologia predominante nas formações montanhosas consiste em granitos, mas também ocorrem migmatitos e gnaisses (BIGARELLA, 1978). Geralmente, acima de $1200 \mathrm{~m}$ snm ocorrem solos pouco profundos, álicos ou distróficos, ricos em matéria orgânica e com baixa CTC e saturação de bases. São comumente encontrados Neossolos Litólicos e Organossolos, também havendo registros de Gleissolos. O acúmulo de matéria orgânica é freqüente, em conseqüência das baixas temperaturas, da alta umidade relativa e precipitação e da esclerofilia de algumas espécies vegetais, que retarda o processo de decomposição (WISNIEWSKI et al., 2005).

O clima é classificado como $\mathrm{Cfb}$, de acordo com Köppen, sendo subtropical, sempre úmido, com a temperatura média do mês mais frio abaixo de $18{ }^{\circ} \mathrm{C}$ e superior a $-3{ }^{\circ} \mathrm{C}$, e a média do mês mais quente inferior a $22^{\circ} \mathrm{C}$. Roderjan; Grodski (1998) observaram temperatura mínima absoluta de $-5^{\circ} \mathrm{C}$, média anual de $13,4{ }^{\circ} \mathrm{C}$ e máxima absoluta de $30^{\circ} \mathrm{C}$ para patamares altomontanos, em ambiente florestal, no Morro do Anhangava, no município de Quatro Barras, Paraná. As precipitações na Serra do Mar são bem distribuídas ao longo do ano e apresentam grande variação em função da topografia. Medições na região litorânea ultrapassam $2000 \mathrm{~mm}$ anuais, e nas encostas da serra os valores chegam a $3500 \mathrm{~mm}$. Já na região do planalto, ultrapassando a cadeia de montanhas, chega-se a $1500 \mathrm{~mm}$ anuais (MAACK, 2002).

A Floresta Ombrófila Densa Atlântica cobre a maior extensão das montanhas, atingindo, em sua formação altomontana, as porções mais elevadas das encostas, acima de $1800 \mathrm{~m}$ snm. Em alguns trechos, nas encostas a oeste, ocorre o ecótono entre esta e a Floresta Ombrófila Mista, com ocorrência de Araucaria angustifolia. Apenas nas porções mais elevadas das montanhas são observados os campos de altitude. Sua ocorrência parece depender de diversos fatores ambientais, como, dentre outros, a altitude, os solos e a exposição aos ventos. Em montanhas de menores altitudes, a formação campestre pode ocorrer pouco acima de $1000 \mathrm{~m}$ snm, nas imediações do cume. Já em montanhas mais altas, os campos de altitude são observados a partir de altitudes mais elevadas, evidenciando a importância de outros fatores ambientais.

Áreas estudadas

Foram amostradas seis subserras, denominadas regionalmente de Serra do Ibitiraquire, Serra da Igreja, Serra da Farinha Seca, Serra da Prata, Serra Gigante e Serra da Pedra Branca do Araraquara (Figura 1). A escolha das áreas de estudo levou em consideração as variações altitudinais, latitudinais, litológicas e geomorfológicas da área de ocorrência dos campos de altitude no Paraná (Tabela 1).

Tabela 1. Características das áreas amostradas na Serra do Mar no Paraná.

Table 1. Characteristics of sampled areas in the sea mountains of Parana.

\begin{tabular}{|c|c|c|c|c|c|}
\hline Serra & $\begin{array}{l}\text { Ponto } \\
\text { culminante } \\
(\mathbf{m} \text { snm) }\end{array}$ & $\begin{array}{l}\text { Localização } \\
\text { (municípios) }\end{array}$ & $\begin{array}{c}\text { Litologia } \\
\text { (Pró-Atlântica, 2002) }\end{array}$ & Características & Proteção por UC \\
\hline Ibitiraquire & 1887 & $\begin{array}{l}\text { Campina } \\
\text { Grande do Sul e } \\
\text { Antonina }\end{array}$ & $\begin{array}{l}\text { Álcali-Granitos (Granito } \\
\text { Graciosa) }\end{array}$ & $\begin{array}{l}\text { Cobertura por campos em cerca de } 790 \text { ha } \\
\text { (RODERJAN, 1999). Ocorrência dos campos a } \\
\text { partir de1350 m snm. Existem } 13 \text { montanhas } \\
\text { com mais de } 1500 \mathrm{~m} \text { snm. }\end{array}$ & $\begin{array}{l}\text { PE do Pico Paraná e } \\
\text { Roberto Ribas Lange }\end{array}$ \\
\hline Igreja & 1376 & Morretes & Álcali-Granitos & $\begin{array}{c}\text { Campos de altitude ocorrem a partir de } 1250 \mathrm{~m} \\
\text { snm. Possui elevações aplainadas. }\end{array}$ & APA de Guaratuba \\
\hline Prata & 1502 & $\begin{array}{l}\text { Morretes, } \\
\text { Paranaguá e } \\
\text { Guaratuba }\end{array}$ & $\begin{array}{l}\text { Complexo Gnáissico- } \\
\text { Migmatítico (Suíte } \\
\text { Granítica Foliada) }\end{array}$ & $\begin{array}{l}\text { Campos de altitude ocorrem a partir de } 1300 \mathrm{~m} \\
\text { snm. Serra isolada na planície litorânea, sem } \\
\text { contato com o planalto. }\end{array}$ & $\begin{array}{l}\text { PN Saint Hilaire- } \\
\text { Lange }\end{array}$ \\
\hline Farinha Seca & 1457 & $\begin{array}{l}\text { Quatro Barras e } \\
\text { Morretes }\end{array}$ & $\begin{array}{l}\text { Álcali-Granitos (Granito } \\
\text { Graciosa) }\end{array}$ & $\begin{array}{l}\text { Campos de altitude ocorrem a partir de } 1300 \mathrm{~m} \\
\text { snm. Possui topos aplainados. }\end{array}$ & PE da Graciosa \\
\hline Gigante & 1069 & $\begin{array}{l}\text { Guaraqueçaba } \\
\text { (PR) e Cananéia } \\
\text { (SP) }\end{array}$ & $\begin{array}{l}\text { Complexo Gnáissico- } \\
\text { Migmatítico (Suíte } \\
\text { Granítica Foliada) } \\
\end{array}$ & $\begin{array}{l}\text { Campos de altitude restritos às proximidades } \\
\text { dos cumes, ocorrendo a partir de } 1050 \mathrm{~m} \text { snm. } \\
\text { Serra isolada na planície litorânea. }\end{array}$ & $\begin{array}{l}\text { APA de Guaraqueçaba } \\
\text { e PE de Jacupiranga } \\
\text { (SP) }\end{array}$ \\
\hline $\begin{array}{l}\text { Pedra Branca do } \\
\text { Araraquara }\end{array}$ & 1222 & Guaratuba & $\begin{array}{l}\text { Complexo Granito- } \\
\text { Gnáissico }\end{array}$ & $\begin{array}{l}\text { Menor trecho de ocorrência dos campos de } \\
\text { altitude dentre as áreas estudadas. Campos de } \\
\text { altitude ocorrem a partir de } 1150 \mathrm{~m} \mathrm{snm} \text {. }\end{array}$ & APA de Guaratuba \\
\hline
\end{tabular}

APA: Área de Proteção Ambiental; PE: Parque Estadual; PN: Parque Nacional.

\section{Levantamento florístico}

O levantamento florístico foi realizado entre agosto de 2002 e agosto de 2004. O esforço amostral variou de acordo com a extensão da formação nas áreas de estudo. A Serra da Igreja foi amostrada entre agosto de 2002 e junho de 2003 em oito fases de campo, totalizando 14 dias de esforço 
de coleta. Na Serra da Farinha Seca, foram realizadas duas fases de campo, uma em dezembro de 2002 e outra em junho de 2003, totalizando seis dias de esforço de coleta. A Serra Gigante foi amostrada em três fases de campo, uma em dezembro de 2002 e as outras em abril e julho de 2003, totalizando nove dias com coleta de dados. Na Serra do Ibitiraquire, foram realizadas oito fases de campo, entre setembro de 2003 e março de 2004, totalizando 17 dias de esforço amostral. A Serra da Prata foi amostrada em duas fases de campo, uma em outubro de 2003 e outra em agosto de 2004, totalizando sete dias de esforço amostral. A Serra da Pedra Branca do Araraquara foi amostrada em apenas uma ocasião, em outubro de 2003, numa fase de campo de dois dias.

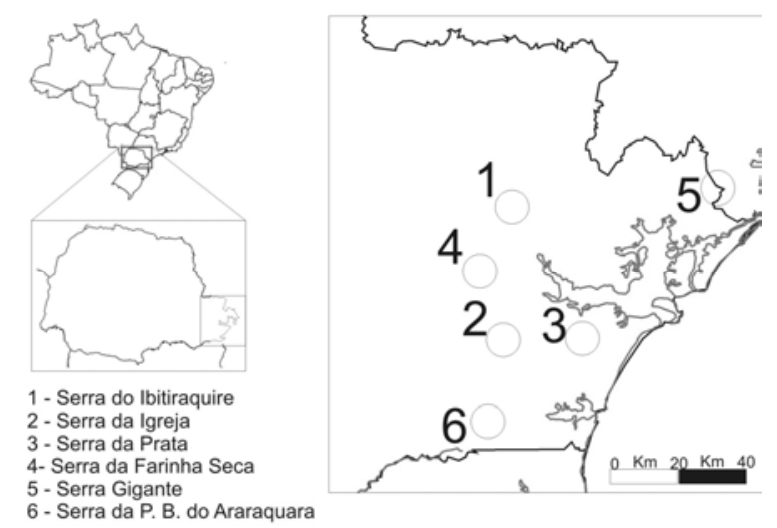

Figura 1. Localização das áreas de estudo no estado do Paraná.

Figure 1. Location of the surveyed areas.

Em cada fase de campo procedeu-se a coleta das espécies vegetais vasculares encontradas férteis na área de ocorrência dos campos de altitude, incluindo aquelas sobre os afloramentos rochosos. Também foram consideradas na amostragem as fisionomias sub-arbustivas e indivíduos arbóreos crescendo isolados, em meio à vegetação campestre ou sub-arbustiva. Em áreas como a Serra do Ibitiraquire e em algumas montanhas da Serra da Farinha Seca, onde, devido ao fluxo constante de montanhistas, existem trilhas consolidadas, fez-se uso destas para a realização das amostragens. Já em áreas mais primitivas, sem trilhas, buscou-se percorrer as áreas de ocorrência através de caminhos que permitissem o acesso à maior extensão possível dos campos. Cabe comentar que, devido à acentuada irregularidade do relevo onde ocorre a vegetação estudada, normalmente não era possível estabelecer e seguir um transecto regular para amostragem.

A determinação das espécies foi realizada com o uso de chaves de identificação, comparação com material de herbários e confirmações com especialistas. Utilizou-se a classificação taxonômica proposta por APG II (2003) para o reconhecimento das famílias de angiospermas, e a proposta por Tryon; Tryon (1982) para as pteridófitas. O material de referência foi incorporado ao acervo do Museu Botânico Municipal de Curitiba (MBM) e as duplicatas foram doadas ao Herbário do Departamento de Botânica da Universidade Federal do Paraná (UPCB) e ao Herbário da Escola de Florestas de Curitiba (EFC).

A análise florística comparativa entre as áreas procedeu-se com o uso do índice de similaridade de Sorensen e com uma análise de agrupamento pelo método de Ward, em função da presença ou ausência de espécies em cada subserra (distância euclidiana ao quadrado foi utilizada como medida métrica). A associação entre a similaridade florística e a distância entre as áreas foi analisada com o uso do coeficiente de correlação de Pearson.

\section{RESULTADOS E DISCUSSÃO}

\section{Composição florística dos campos de altitude na Serra do Mar paranaense}

Foram encontradas 280 espécies vegetais vasculares nos seis trechos estudados na Serra do Mar paranaense (Tabela 2), pertencentes a 73 famílias botânicas, sendo 63 angiospermas (260 espécies), oito pteridófitas (18 espécies) e duas gimnospermas ( 2 espécies). Esse total de espécies é apenas uma primeira 
aproximação da riqueza florística dos campos de altitude na Serra do Mar no Paraná. A lista apresentada não incorporou espécies previamente registradas em herbários e espécies encontradas em outros trabalhos que não tenham sido detectadas pelo presente estudo. Em outro estudo, sobre estrutura da vegetação, realizado nas mesmas áreas e pelos mesmos autores, foram encontrados outros 40 morfotipos (dados ainda não publicados), cuja identificação ainda não foi possível em função da ausência de amostras férteis.

Tabela 2. Espécies vasculares dos campos de altitude na Serra do Mar paranaense. Legenda: Forma biológica: ARB: arbusto; ARV: árvore; EPI: epífita; HER: herbácea; TRE: trepadeira. Áreas de estudo: IBI: Serra do Ibitiraquire; IGR: Serra da Igreja; PRA: Serra da Prata; FAS: Serra da Farinha Seca; GIG: Serra Gigante; PBA: Serra da Pedra Branca do Araraquara. Coletor: AYM: Alan Yukio Mocochinski; MBS: Maurício Bergamini Scheer; RTP: Ruddy Thomas Proença.

Table 2. List of high-altitude grasslands species in Sea Mountain Range in Southern Brazil. Legend: Life-form: ARB: shrub; ARV: tree; EPI: epiphyte; HER: herbaceous; TRE: climbers. Investigated areas: IBI: Serra do Ibitiraquire; IGR: Serra da Igreja; PRA: Serra da Prata; FAS: Serra da Farinha Seca; GIG: Serra Gigante; PBA: Serra da Pedra Branca do Araraquara. Sample: AYM: Alan Yukio Mocochinski; MBS: Maurício Bergamini Scheer; RTP: Ruddy Thomas Proença.

\begin{tabular}{|c|c|c|c|c|c|c|c|c|c|}
\hline Família & Espécie & Forma & IBI & IGR & PRA & FAS & GIG & PBA & $\begin{array}{l}\text { Coletor / } \\
\text { Amostra }\end{array}$ \\
\hline \multicolumn{10}{|c|}{ DIVISÃO ANGIOSPERMAE } \\
\hline Alstroemeriaceae & Alstroemeria amabilis M.C. Assis & HER & $\mathrm{X}$ & $\mathrm{X}$ & $\mathrm{X}$ & $\mathrm{X}$ & & & AYM 162 \\
\hline Amaryllidaceae & Hippeastrum illustre (Vell.) Dutilh & HER & $\mathrm{X}$ & $\mathrm{X}$ & $\mathrm{X}$ & & $\mathrm{X}$ & & MBS 532 \\
\hline \multirow[t]{2}{*}{ Apiaceae } & Eryngium koehneanum Urban & HER & $\mathrm{X}$ & & & $\mathrm{X}$ & & $\mathrm{X}$ & MBS 1 \\
\hline & Eryngium scirpinum Cham. \& Schltdl. & HER & $\mathrm{X}$ & & & & & & MBS 638 \\
\hline \multirow[t]{7}{*}{ Apocynaceae } & Aspidosperma pyricollum Müll. Arg. & ARV & & & & & $\mathrm{X}$ & & AYM 159 \\
\hline & Mandevilla atroviolacea (Stadelm.) R.E. Woodson & TRE & $\mathrm{X}$ & & & & $\mathrm{X}$ & & MBS 226 \\
\hline & Mandevilla immaculata R.E. Woodson & TRE & $\mathrm{X}$ & $\mathrm{X}$ & & & & & AYM 35 \\
\hline & Orthosia dusenii (Malme) Fontella & TRE & $\mathrm{X}$ & $\mathrm{X}$ & $\mathrm{X}$ & & & & MBS 511 \\
\hline & Oxypetalum sublanatum Malme & HER & $\mathrm{X}$ & & & & & & MBS 120 \\
\hline & Oxypetalum sp. & TRE & & $\mathrm{X}$ & $\mathrm{X}$ & $\mathrm{X}$ & & $\mathrm{X}$ & AYM 28 \\
\hline & Apocynaceae indeterminada & TRE & $\mathrm{X}$ & $\mathrm{X}$ & & $\mathrm{X}$ & $\mathrm{X}$ & $\mathrm{X}$ & MBS 761 \\
\hline \multirow[t]{3}{*}{ Aquifoliaceae } & Ilex chamaedryfolia Reissek & ARV & $\mathrm{X}$ & $\mathrm{X}$ & & & & & AYM 157 \\
\hline & Ilex microdonta Reissek & ARV & $\mathrm{X}$ & $\mathrm{X}$ & $\mathrm{X}$ & $\mathrm{X}$ & & $\mathrm{X}$ & AYM 32 \\
\hline & Ilex theazans Martius & ARV & $\mathrm{X}$ & & & & $\mathrm{X}$ & & MBS 528 \\
\hline Araceae & Philodendron glaziovii Hook. f. & HER & & & & & $\mathrm{X}$ & & MBS 616 \\
\hline Araliaceae & Hydrocotyle quinqueloba Ruiz \& Pav. & HER & $\mathrm{X}$ & $\mathrm{X}$ & & & & & MBS 530 \\
\hline \multirow[t]{16}{*}{ Asteraceae } & $\begin{array}{l}\text { Achyrocline satureioides (Lam.) DC. } \\
\text { Austroeupatorium neglectum (B.L. Rob.) R.M. King \& }\end{array}$ & HER & $\mathrm{X}$ & & & & & & MBS 44 \\
\hline & H. Rob. & ARB & $\mathrm{X}$ & & & $\mathrm{X}$ & $\mathrm{X}$ & & MBS 619 \\
\hline & Baccharis angusticeps Dusén & ARB & $\mathrm{X}$ & $\mathrm{X}$ & $\mathrm{X}$ & $\mathrm{X}$ & & $\mathrm{X}$ & MBS 290 \\
\hline & Baccharis aracatubensis Teodoro \& Hatschbach & $\mathrm{ARB}$ & & $\mathrm{X}$ & & & & & MBS 625 \\
\hline & Baccharis brachylaenoides DC. var. brachylaenoides & $\mathrm{ARB}$ & $\mathrm{X}$ & & & $\mathrm{X}$ & & & MBS 408 \\
\hline & $\begin{array}{l}\text { Baccharis brachylaenoides DC. } \\
\text { Barroso } \\
\text { var. polycephala (Malag.) G.M. }\end{array}$ & ARB & $\mathrm{X}$ & & & & $\mathrm{X}$ & & AYM 3 \\
\hline & Baccharis cassinifolia DC. & ARB & $\mathrm{X}$ & $\mathrm{X}$ & $\mathrm{X}$ & $\mathrm{X}$ & & $\mathrm{X}$ & MBS 57 \\
\hline & Baccharis curitybensis Heering \& Dusén & HER & $\mathrm{X}$ & & $\mathrm{X}$ & $\mathrm{X}$ & & $\mathrm{X}$ & AYM 129 \\
\hline & Baccharis erioclada DC. & HER & $\mathrm{X}$ & & & & & & MBS 45 \\
\hline & Baccharis illinita DC. & HER & $\mathrm{X}$ & & & & & & AYM 378 \\
\hline & Baccharis intermixta Gardn. & ARB & $\mathrm{X}$ & & & & & & $\mathrm{AYM} \mathrm{s} / \mathrm{n}^{\circ}$ \\
\hline & Baccharis leucocephala Dusén & HER & $\mathrm{X}$ & & & & & & MBS 632 \\
\hline & Baccharis oreophila Malme & $\mathrm{ARB}$ & $\mathrm{X}$ & & & & & & AYM s $/ n^{\circ}$ \\
\hline & Baccharis organensis Baker & ARB & $\mathrm{X}$ & & & $\mathrm{X}$ & & $\mathrm{X}$ & MBS 423 \\
\hline & Baccharis platypoda DC. & HER & $\mathrm{X}$ & & & & & & MBS 6 \\
\hline & Baccharis retusa DC. & $\mathrm{ARB}$ & $\mathrm{X}$ & & & & & & MBS 70 \\
\hline
\end{tabular}




\begin{tabular}{|c|c|c|c|c|c|c|c|c|c|}
\hline Família & Espécie & Forma & IBI & IGR & PRA & FAS & GIG & PBA & $\begin{array}{l}\text { Coletor/ } \\
\text { Amostra } \\
\end{array}$ \\
\hline & Baccharis tarchonanthoides DC. & ARB & $\mathrm{X}$ & & & & & & AYM 257 \\
\hline & Baccharis cf. trimera (Less.) DC. & ARB & $\mathrm{X}$ & $\mathrm{X}$ & $\mathrm{X}$ & $\mathrm{X}$ & $\mathrm{X}$ & $\mathrm{X}$ & MBS 415 \\
\hline & Baccharis uncinella DC. & ARB & $\mathrm{X}$ & & & & & & MBS 626 \\
\hline & Baccharis sp. & ARB & $\mathrm{X}$ & $\mathrm{X}$ & & & & $\mathrm{X}$ & MBS 414 \\
\hline & Campovassouria cruciata (Vell.) R.M. King \& H. Rob. & $\mathrm{ARB}$ & $\mathrm{X}$ & & & & & & MBS 47 \\
\hline & Critoniopsis quinqueflora (Less.) H. Rob. & HER & & $\mathrm{X}$ & $\mathrm{X}$ & $\mathrm{X}$ & & & AYM 255 \\
\hline & Dasyphyllum spinescens (Less.) Cabrera & ARB & $\mathrm{X}$ & & $\mathrm{X}$ & $\mathrm{X}$ & & & AYM 259 \\
\hline & Dendrophorbium limosum C. Jeffrey & HER & $\mathrm{X}$ & $\mathrm{X}$ & $\mathrm{X}$ & $\mathrm{X}$ & $\mathrm{X}$ & $\mathrm{X}$ & MBS 13 \\
\hline & Erigeron catarinensis Cabrera & HER & $\mathrm{X}$ & & & & & & MBS 628 \\
\hline & Graphistylis serrana (Zardini) B. Nord. & HER & $\mathrm{X}$ & $\mathrm{X}$ & $\mathrm{X}$ & $\mathrm{X}$ & & & MBS 59 \\
\hline & Grazielia cf. serrata (Spreng.) R.M. King \& H. Rob. & HER & $\mathrm{X}$ & $\mathrm{X}$ & & $\mathrm{X}$ & & & AYM 256 \\
\hline & Heterocondylus alatus (Vell.) R.M. King \& H. Rob. & $\mathrm{ARB}$ & $\mathrm{X}$ & $\mathrm{X}$ & $\mathrm{X}$ & $\mathrm{X}$ & & & MBS 407 \\
\hline & Hypochaeris catharinensis Cabrera & HER & $\mathrm{X}$ & & & & & & $\mathrm{AYM} s / \mathrm{n}^{\circ}$ \\
\hline & Mikania campanulata Gardn. & TRE & $\mathrm{X}$ & & $\mathrm{X}$ & & $\mathrm{X}$ & $\mathrm{X}$ & MBS 14 \\
\hline & Mikania clematidifolia Dusén & TRE & $\mathrm{X}$ & $\mathrm{X}$ & & $\mathrm{X}$ & & & MBS 622 \\
\hline & Mikania involucrata Hook. \& Arn. & TRE & & & & & $\mathrm{X}$ & & AYM 258 \\
\hline & Mikania lanuginosa DC. & TRE & $\mathrm{X}$ & $\mathrm{X}$ & $\mathrm{X}$ & & $\mathrm{X}$ & $\mathrm{X}$ & MBS 621 \\
\hline & Mikania lindbergii Baker & TRE & & & & & $\mathrm{X}$ & $\mathrm{X}$ & AYM 20 \\
\hline & Mikania paranensis Dusén & TRE & $\mathrm{X}$ & & & $\mathrm{X}$ & & & MBS 636 \\
\hline & Pentacalia desiderabilis (Vell.) Cuatrec. & TRE & $\mathrm{X}$ & $\mathrm{X}$ & $\mathrm{X}$ & $\mathrm{X}$ & & & AYM 254 \\
\hline & Piptocarpha densifolia Dusén ex G.L. Smith & ARV & $\mathrm{X}$ & $\mathrm{X}$ & $\mathrm{X}$ & & $\mathrm{X}$ & $\mathrm{X}$ & $\mathrm{M}^{2} \quad 27$ \\
\hline & Stevia claussenii Sch. Bip. ex Baker & HER & $\mathrm{X}$ & $\mathrm{X}$ & & & & & MBS 248 \\
\hline & Symphyopappus cuneatus Sch. Bip. & ARB & $\mathrm{X}$ & $\mathrm{X}$ & $\mathrm{X}$ & & & $\mathrm{X}$ & MBS 123 \\
\hline & Symphyopappus lymansmithii B.L. Rob. & ARB & & $\mathrm{X}$ & $\mathrm{X}$ & & & $\mathrm{X}$ & MBS 624 \\
\hline & Trichocline catharinensis Cabrera var. discolor Cabrera & HER & $\mathrm{X}$ & & & & & & AYM 94 \\
\hline & Trixis brasiliensis $(\mathrm{L}.) \mathrm{DC}$. & HER & $\mathrm{X}$ & $\mathrm{X}$ & $\mathrm{X}$ & $\mathrm{X}$ & & $\mathrm{X}$ & AYM 252 \\
\hline & Trixis lessingii DC. & HER & $\mathrm{X}$ & & & & & & MBS 124 \\
\hline & Verbesina glabrata Hook. \& Arn. & HER & & $\mathrm{X}$ & $\mathrm{X}$ & $\mathrm{X}$ & & & MBS 203 \\
\hline & Vernonanthura montevidensis (Spreng.) H. Rob. & $\mathrm{ARB}$ & $\mathrm{X}$ & $\mathrm{X}$ & $\mathrm{X}$ & $\mathrm{X}$ & & $\mathrm{X}$ & MBS 87 \\
\hline Berberidaceae & Berberis laurina Billb. & $\mathrm{ARB}$ & $\mathrm{X}$ & & & & & & MBS 121 \\
\hline \multirow[t]{4}{*}{ Bignoniaceae } & $\begin{array}{l}\text { Anemopaegma chamberlaynii (Sims) Bureau \& K. } \\
\text { Schum. }\end{array}$ & TRE & $\mathrm{X}$ & $\mathrm{X}$ & $\mathrm{X}$ & & & $\mathrm{X}$ & AYM 163 \\
\hline & Anemopaegma prostratum DC. & TRE & & & & & $\mathrm{X}$ & & MBS 535 \\
\hline & Anemopaegma sp. & TRE & & & & & & $\mathrm{X}$ & AYM 373 \\
\hline & Tabebuia catarinensis A. Gentry & ARV & $\mathrm{X}$ & $\mathrm{X}$ & $\mathrm{X}$ & $\mathrm{X}$ & $\mathrm{X}$ & & AYM 6 \\
\hline \multirow[t]{7}{*}{ Bromeliaceae } & Dyckia reitzii L.B. Smith & HER & $\mathrm{X}$ & & & & & & \\
\hline & Pitcairnia flammea Lindl. var. floccosa L.B. Sm. & HER & $\mathrm{X}$ & & $\mathrm{X}$ & & $\mathrm{X}$ & & MBS 536 \\
\hline & Quesnelia imbricata L.B. Sm. & HER & $\mathrm{X}$ & & & & $\mathrm{X}$ & & AYM 168 \\
\hline & Vriesea altodaserrae L.B. Sm. & HER & & & & & $\mathrm{X}$ & & AYM 167 \\
\hline & Vriesea cf. hoehneana L.B. Sm. & HER & $\mathrm{X}$ & $\mathrm{X}$ & $\mathrm{X}$ & & & $\mathrm{X}$ & $\mathrm{AYM} \mathrm{s} / \mathrm{n}^{\circ}$ \\
\hline & Vriesea platynema var. variegata Gaudich. & HER & $\mathrm{X}$ & $\mathrm{X}$ & $\mathrm{X}$ & $\mathrm{X}$ & $\mathrm{X}$ & $\mathrm{X}$ & AYM 166 \\
\hline & Vriesea $\mathrm{sp}$. & HER & & & & & $\mathrm{X}$ & & AYM 44 \\
\hline Cactaceae & Cactaceae indeterminada & HER & $\mathrm{X}$ & & & & & & MBS 757 \\
\hline \multirow[t]{2}{*}{ Campanulaceae } & Lobelia camporum Pohl & HER & $\mathrm{X}$ & & & & & & MBS 131 \\
\hline & Siphocampylus eichleri Kanitz & HER & $\mathrm{X}$ & & & & & & MBS 540 \\
\hline Cardiopteridaceae & Citronella paniculata (Mart.) Howard & ARV & & & & $\mathrm{X}$ & & & AYM 182 \\
\hline Celastraceae & Maytenus urbaniana Loes. & ARV & $\mathrm{X}$ & & & & & & AYM 170 \\
\hline Chlorantaceae & Hedyosmum brasiliense Miq. & ARV & & & & & $\mathrm{X}$ & & MBS 544 \\
\hline \multirow[t]{2}{*}{ Clethraceae } & Clethra scabra Pers. & ARV & & & & $\mathrm{X}$ & & & AYM 172 \\
\hline & Clethra uleana Sleumer & $\mathrm{ARV}$ & $\mathrm{X}$ & $\mathrm{X}$ & $\mathrm{X}$ & $\mathrm{X}$ & $\mathrm{X}$ & $\mathrm{X}$ & MBS 353 \\
\hline Clusiaceae & Clusia criuva Cambess. & $\mathrm{ARB}$ & & & & & $\mathrm{X}$ & & MBS 224 \\
\hline Cornaceae & Griselinia ruscifolia (Clos) Taub. & TRE & $\mathrm{X}$ & $\mathrm{X}$ & $\mathrm{X}$ & & $\mathrm{X}$ & & AYM 46 \\
\hline Cunoniaceae & Weinmannia humilis Engl. & ARV & $\mathrm{X}$ & $\mathrm{X}$ & $\mathrm{X}$ & $\mathrm{X}$ & & & MBS 16 \\
\hline
\end{tabular}




\begin{tabular}{|c|c|c|c|c|c|c|c|c|c|}
\hline Família & Espécie & Forma & IBI & IGR & PRA & FAS & GIG & PBA & $\begin{array}{l}\text { Coletor / } \\
\text { Amostra }\end{array}$ \\
\hline \multirow[t]{16}{*}{ Cyperaceae } & Bulbostylis consanguinea Nees & HER & $\mathrm{X}$ & & & & $\mathrm{X}$ & & \\
\hline & Cyperus sp. & HER & $\mathrm{X}$ & & & & & & AYM 295 \\
\hline & Lagenocarpus triquetrus (Boeck.) Kuntze & HER & $\mathrm{X}$ & $\mathrm{X}$ & $\mathrm{X}$ & $\mathrm{X}$ & $\mathrm{X}$ & $\mathrm{X}$ & MBS 555 \\
\hline & Machaerina austrobrasiliensis M.T. Strong & HER & $\mathrm{X}$ & $\mathrm{X}$ & $\mathrm{X}$ & $\mathrm{X}$ & $\mathrm{X}$ & $\mathrm{X}$ & AYM 128 \\
\hline & Rhynchospora exaltata Kunth & HER & $\mathrm{X}$ & $\mathrm{X}$ & $\mathrm{X}$ & $\mathrm{X}$ & $\mathrm{X}$ & & AYM 122 \\
\hline & Rhynchospora polyantha Steud. & HER & $\mathrm{X}$ & & & & & & $\mathrm{AYM} \mathrm{s} / \mathrm{n}^{\circ}$ \\
\hline & Rhynchospora sp. 1 & HER & $\mathrm{X}$ & $\mathrm{X}$ & & & & & MBS 673 \\
\hline & Rhynchospora sp. 2 & HER & & & & & & $\mathrm{X}$ & MBS 674 \\
\hline & Rhynchospora sp. 3 & HER & & & $\mathrm{X}$ & & & & MBS 490 \\
\hline & Rhynchospora sp. 4 & HER & $\mathrm{X}$ & & & & & & AYM 297 \\
\hline & Rhynchospora sp. 5 & HER & $\mathrm{X}$ & & & & $\mathrm{X}$ & $\mathrm{X}$ & AYM 156 \\
\hline & Scleria cf. balansae Maury & HER & $\mathrm{X}$ & & & & & & $\mathrm{AYM} s / \mathrm{n}^{\circ}$ \\
\hline & Cyperaceae indeterminada 1 & HER & $\mathrm{X}$ & & & & & & RTP 103 \\
\hline & Cyperaceae indeterminada 2 & HER & & $\mathrm{X}$ & & $\mathrm{X}$ & & & AYM 296 \\
\hline & Cyperaceae indeterminada 3 & HER & & $\mathrm{X}$ & & & & & AYM 103 \\
\hline & Cyperaceae indeterminada 4 & HER & & & & & & $\mathrm{X}$ & MBS 110 \\
\hline Dioscoreaceae & Dioscorea sanpaulensis R. Kunth & TRE & $\mathrm{X}$ & $\mathrm{X}$ & & & $\mathrm{X}$ & & AYM 174 \\
\hline Droseraceae & Drosera montana A.St.-Hil. & HER & $\mathrm{X}$ & $\mathrm{X}$ & $\mathrm{X}$ & & & & AYM 175 \\
\hline \multirow[t]{14}{*}{ Ericaceae } & $\begin{array}{l}\text { Agarista niederleinii (Sleumer) Judd var. acutifolia } \\
\text { W.S. Judd }\end{array}$ & $\mathrm{ARB}$ & $\mathrm{X}$ & & $\mathrm{X}$ & & & & AYM 177 \\
\hline & Agarista niederleinii (Sleumer) Judd var. niederleinii & ARB & & $\mathrm{X}$ & & & & $\mathrm{X}$ & MBS 547 \\
\hline & Agarista pulchella Cham. ex G. Don & ARB & $\mathrm{X}$ & & & & & & MBS 548 \\
\hline & $\begin{array}{l}\text { Gaultheria itatiaiae Wawra } \\
\text { Gaultheria serrata K-Gouv. var. organensis (Meisn.) }\end{array}$ & ARB & $\mathrm{X}$ & & $\mathrm{X}$ & & & & AYM 176 \\
\hline & Luteyn & ARB & $\mathrm{X}$ & $\mathrm{X}$ & $\mathrm{X}$ & $\mathrm{X}$ & & & AYM 48 \\
\hline & Gaultheria sp. 1 & ARB & $\mathrm{X}$ & & & & & & MBS 754 \\
\hline & Gaultheria sp. 2 & $\mathrm{ARB}$ & $\mathrm{X}$ & & & & & & AYM 372 \\
\hline & Gaylussacia arassatubensis R.R. Silva \& Cervi & ARB & $\mathrm{X}$ & & & & & & MBS 378 \\
\hline & Gaylussacia brasiliensis (Spr.) Meissn. var. brasiliensis & $\mathrm{ARB}$ & $\mathrm{X}$ & $\mathrm{X}$ & $\mathrm{X}$ & $\mathrm{X}$ & $\mathrm{X}$ & $\mathrm{X}$ & MBS 77 \\
\hline & Gaylussacia caratuvensis R.R. Silva \& Cervi & $\mathrm{ARB}$ & $\mathrm{X}$ & & & & & & MBS 753 \\
\hline & Gaylussacia sp. & ARB & $\mathrm{X}$ & $\mathrm{X}$ & $\mathrm{X}$ & & & $\mathrm{X}$ & MBS 394 \\
\hline & Ericaceae indeterminada 1 & ARB & & $\mathrm{X}$ & $\mathrm{X}$ & & & $\mathrm{X}$ & MBS 752 \\
\hline & Ericaceae indeterminada 2 & ARB & & & & & & $\mathrm{X}$ & AYM 371 \\
\hline & Ericaceae indeterminada 3 & ARV & $\mathrm{X}$ & & & & & & MBS 755 \\
\hline \multirow[t]{2}{*}{ Eriocaulaceae } & Eriocaulon ligulatum (Vell.) L.B. Smith & HER & $\mathrm{X}$ & $\mathrm{X}$ & $\mathrm{X}$ & & & & AYM 51 \\
\hline & Eriocaulon $\mathrm{sp}$. & HER & $\mathrm{X}$ & & & & & & AYM 370 \\
\hline Escalloniaceae & Escallonia laevis (Vell.) Sleum. & $\mathrm{ARB}$ & $\mathrm{X}$ & & & & & & MBS 247 \\
\hline \multirow[t]{2}{*}{ Euphorbiaceae } & Croton splendidus Mart. ex Baill. & $\mathrm{ARB}$ & $\mathrm{X}$ & $\mathrm{X}$ & $\mathrm{X}$ & $\mathrm{X}$ & & & MBS 79 \\
\hline & Pera glabrata $($ Schott) Baill. & ARB & $\mathrm{X}$ & & & & $\mathrm{X}$ & & AYM 179 \\
\hline $\begin{array}{l}\text { Fabaceae - } \\
\text { Caesalpinioideae } \\
\end{array}$ & $\begin{array}{l}\text { Senna organensis (Glaz. ex Harms) Irwin \& Barneby } \\
\text { var. extratropica Irwin \& Barneby }\end{array}$ & ARV & $\mathrm{X}$ & & $\mathrm{X}$ & $\mathrm{X}$ & & $\mathrm{X}$ & AYM 222 \\
\hline Fabaceae - Faboideae & Dalbergia frutescens (Vellozo) Britton & $\mathrm{ARB}$ & $\mathrm{X}$ & & & & & & AYM 58 \\
\hline Fabaceae - & Inga barbata Bentham & $\mathrm{ARB}$ & $\mathrm{X}$ & & & & & & MBS 592 \\
\hline \multirow[t]{6}{*}{ Mimosoideae } & Mimosa atlantica Barneby & $\mathrm{ARB}$ & & & & & & $\mathrm{X}$ & MBS 590 \\
\hline & Mimosa congestifolia Burkart & ARB & $\mathrm{X}$ & & & $\mathrm{X}$ & & & MBS 355 \\
\hline & Mimosa eurystegia Barneby & TRE & $\mathrm{X}$ & & & & & & $\mathrm{AYM} s / \mathrm{n}^{\circ}$ \\
\hline & Mimosa prionopus Barneby & TRE & $\mathrm{X}$ & & & & & & MBS 428 \\
\hline & Mimosa pseudocalosa Burkart & TRE & $\mathrm{X}$ & & & & & & AYM 221 \\
\hline & Mimosa tucumensis Barneby ex Ribas & TRE & $\mathrm{X}$ & & & & & & MBS 591 \\
\hline Gentianaceae & Macrocarpaea rubra Malme & HER & & $\mathrm{X}$ & & & $\mathrm{X}$ & & MBS 115 \\
\hline \multirow[t]{2}{*}{ Gesneriaceae } & Nematanthus sp. & HER & $\mathrm{X}$ & $\mathrm{X}$ & & & $\mathrm{X}$ & & \\
\hline & Sinningia aff. magnifica (Otto \& A. Dietr.) Wiehle & HER & $\mathrm{X}$ & & & & $\mathrm{X}$ & & AYM 369 \\
\hline \multirow[t]{2}{*}{ Iridaceae } & Alophia geniculata Klatt & HER & $\mathrm{X}$ & $\mathrm{X}$ & $\mathrm{X}$ & $\mathrm{X}$ & & & AYM 183 \\
\hline & Neomarica sp. & HER & & & & & $\mathrm{X}$ & & MBS 759 \\
\hline
\end{tabular}

FLORESTA, Curitiba, PR, v. 38, n. 4, p. 625-640, out./dez. 2008. 


\begin{tabular}{|c|c|c|c|c|c|c|c|c|c|}
\hline Família & Espécie & Forma & IBI & IGR & PRA & FAS & GIG & PBA & $\begin{array}{l}\text { Coletor/ } \\
\text { Amostra } \\
\end{array}$ \\
\hline & Sisyrinchium vaginatum Spreng. & HER & $\mathrm{X}$ & & $\mathrm{X}$ & & $\mathrm{X}$ & & MBS 553 \\
\hline Juncaceae & Juncus sp. & HER & $\mathrm{X}$ & & & & & & \\
\hline \multirow[t]{4}{*}{ Lamiaceae } & Hesperozygis nitida (Bentham) Epling & ARB & $\mathrm{X}$ & & & & & & MBS 130 \\
\hline & Hesperozygis rhododon Epling & ARB & $\mathrm{X}$ & $\mathrm{X}$ & $\mathrm{X}$ & $\mathrm{X}$ & & $\mathrm{X}$ & MBS 291 \\
\hline & Hesperozygis sp. & HER & $\mathrm{X}$ & & & & & & AYM 367 \\
\hline & Vitex sp. & ARV & $\mathrm{X}$ & & & & & & \\
\hline \multirow[t]{6}{*}{ Lauraceae } & Ocotea bicolor Vattimo-Gil & ARB & $\mathrm{X}$ & & & & & & MBS 427 \\
\hline & Ocotea porosa (Nees \& C. Mart.) Barroso & ARV & & $\mathrm{X}$ & $\mathrm{X}$ & & $\mathrm{X}$ & & AYM 56 \\
\hline & Ocotea pulchella (Nees) Mez & ARV & & $\mathrm{X}$ & & $\mathrm{X}$ & & & MBS 284 \\
\hline & Ocotea tristis (Nees \& Martius) Mez & ARV & $\mathrm{X}$ & $\mathrm{X}$ & $\mathrm{X}$ & $\mathrm{x}$ & $\mathrm{X}$ & $\mathrm{X}$ & MBS 584 \\
\hline & Persea alba Nees & ARV & $\mathrm{X}$ & & & $\mathrm{X}$ & & & AYM 213 \\
\hline & Persea pyrifolia Nees & ARV & $\mathrm{X}$ & & & & & & AYM 57 \\
\hline \multirow[t]{3}{*}{ Lentibulariaceae } & Utricularia reniformis A. St.-Hil. & HER & $\mathrm{X}$ & $\mathrm{X}$ & $\mathrm{X}$ & $\mathrm{X}$ & $\mathrm{X}$ & $\mathrm{X}$ & MBS 491 \\
\hline & Utricularia sp. 1 & HER & $\mathrm{X}$ & & & & & & \\
\hline & Utricularia sp. 2 & HER & $\mathrm{X}$ & & & & & & MBS 587 \\
\hline Liliaceae & Lilium regale E.H. Wilson & HER & $\mathrm{X}$ & & & & & & AYM 218 \\
\hline Loranthaceae & Struthanthus complexus Eichler & TRE & $\mathrm{X}$ & & & & & $\mathrm{X}$ & MBS 588 \\
\hline \multirow[t]{17}{*}{ Melastomataceae } & Leandra calvescens (Triana) Cogn. & HER & $\mathrm{X}$ & & & & & & AYM 277 \\
\hline & Leandra cordigera Cogn. & HER & $\mathrm{X}$ & $\mathrm{X}$ & $\mathrm{X}$ & $\mathrm{X}$ & & & AYM 282 \\
\hline & Leandra cf. quinquedentata (DC.) Cogn. & ARB & $\mathrm{X}$ & $\mathrm{X}$ & $\mathrm{X}$ & $\mathrm{X}$ & $\mathrm{X}$ & $\mathrm{X}$ & AYM 281 \\
\hline & Leandra cf. sublanata Cogn. & ARB & $\mathrm{X}$ & & & $\mathrm{X}$ & & & AYM 275 \\
\hline & Leandra cf. sulfurea (Naudin) Cogn. & ARB & $\mathrm{X}$ & & & & & & MBS 658 \\
\hline & Leandra sp. & HER & $\mathrm{X}$ & & & & & & AYM 274 \\
\hline & Miconia lymanii Wurdack & ARB & $\mathrm{X}$ & $\mathrm{X}$ & $\mathrm{X}$ & $\mathrm{X}$ & $\mathrm{X}$ & $\mathrm{X}$ & AYM 62 \\
\hline & Miconia ramboii Brade & $\mathrm{ARB}$ & $\mathrm{X}$ & & & & & & MBS 431 \\
\hline & Miconia sellowiana Naudin & ARV & $\mathrm{X}$ & & & & & & MBS 654 \\
\hline & Miconia sp. & $\mathrm{ARB}$ & $\mathrm{X}$ & & & & & & MBS 656 \\
\hline & Tibouchina dubia (Cham.) Cogn. & ARB & $\mathrm{X}$ & $\mathrm{X}$ & $\mathrm{X}$ & $\mathrm{X}$ & & $\mathrm{X}$ & AYM 284 \\
\hline & Tibouchina hospita (DC.) Cogn. & ARB & $\mathrm{X}$ & $\mathrm{X}$ & $\mathrm{X}$ & $\mathrm{X}$ & & & MBS 661 \\
\hline & Tibouchina marumbiensis Wurdack & ARV & & & & & $\mathrm{X}$ & & MBS 325 \\
\hline & Tibouchina reitzii Brade & ARV & $\mathrm{X}$ & & & $\mathrm{X}$ & & $\mathrm{X}$ & MBS 286 \\
\hline & Tibouchina sp. 1 & $\mathrm{ARB}$ & $\mathrm{X}$ & & & & & & $\mathrm{AYM} \mathrm{s} / \mathrm{n}^{\circ}$ \\
\hline & Tibouchina sp. 2 & $\mathrm{ARB}$ & $\mathrm{X}$ & & & & & & AYM 271 \\
\hline & Melastomataceae indeterminada & $\mathrm{ARB}$ & $\mathrm{X}$ & & & & & & AYM 273 \\
\hline Myrsinaceae & Myrsine altomontana M.F. Freitas \& L.S. Kinoshita & ARV & $\mathrm{X}$ & $\mathrm{X}$ & $\mathrm{X}$ & $\mathrm{X}$ & & $\mathrm{X}$ & AYM 226 \\
\hline \multirow[t]{13}{*}{ Myrtaceae } & Eugenia neomyrtifolia Sobral & ARB & & & $\mathrm{X}$ & $\mathrm{X}$ & & & AYM 126 \\
\hline & Gomidesia sellowiana O. Berg & ARV & $\mathrm{X}$ & $\mathrm{X}$ & $\mathrm{X}$ & $\mathrm{X}$ & $\mathrm{X}$ & $\mathrm{X}$ & AYM 320 \\
\hline & Myrceugenia alpigena (DC.) Landrum & $\mathrm{ARB}$ & $\mathrm{X}$ & & & & & & AYM 264 \\
\hline & Myrceugenia franciscensis (O. Berg) Landrum & $\mathrm{ARB}$ & $\mathrm{X}$ & & $\mathrm{X}$ & $\mathrm{X}$ & & & AYM 130 \\
\hline & Myrceugenia pilotantha (Kiaersk.) Landrum & ARV & & & $\mathrm{X}$ & & & $\mathrm{X}$ & AYM 303 \\
\hline & Myrcia breviramis (O. Berg) C.D. Legrand & ARV & $\mathrm{X}$ & $\mathrm{X}$ & $\mathrm{X}$ & & $\mathrm{X}$ & & MBS 65 \\
\hline & Myrcia obtecta Kiaersk. & ARV & $\mathrm{X}$ & & $\mathrm{X}$ & $\mathrm{X}$ & $\mathrm{X}$ & $\mathrm{X}$ & AYM 263 \\
\hline & Myrcia richardiana (O. Berg) Kiaersk. & ARV & & & & $\mathrm{X}$ & $\mathrm{X}$ & & MBS 496 \\
\hline & Myrciaria tenella (DC.) O. Berg & ARV & $\mathrm{X}$ & $\mathrm{X}$ & $\mathrm{X}$ & $\mathrm{X}$ & & $\mathrm{X}$ & AYM 269 \\
\hline & Pimenta pseudocaryophyllus (Gomes) Landrum & ARV & & & & & $\mathrm{X}$ & $\mathrm{X}$ & AYM 270 \\
\hline & Psidium spathulatum Mattos & ARV & & & & $\mathrm{X}$ & & & AYM 261 \\
\hline & Siphoneugena reitzii C.D. Legrand & ARV & $\mathrm{X}$ & $\mathrm{X}$ & $\mathrm{X}$ & $\mathrm{X}$ & $\mathrm{X}$ & $\mathrm{X}$ & AYM 123 \\
\hline & Myrtaceae indeterminada & ARV & & & & & & $\mathrm{X}$ & AYM 286 \\
\hline Ochnaceae & Ouratea vaccinioides Engl. & ARV & $\mathrm{X}$ & & & & $\mathrm{X}$ & & AYM 186 \\
\hline Onagraceae & Fuchsia regia (Vell) Munz ssp. serrae P.E. Berry & $\mathrm{ARB}$ & $\mathrm{X}$ & $\mathrm{X}$ & $\mathrm{X}$ & & & & AYM 144 \\
\hline \multirow[t]{2}{*}{ Orchidaceae } & Cleistes brasiliensis (Barbosa Rodr.) Schlechter & HER & $\mathrm{X}$ & $\mathrm{X}$ & & $\mathrm{X}$ & & & AYM 187 \\
\hline & Epidendrum ellipticum Grah. & HER & $\mathrm{X}$ & $\mathrm{X}$ & & & $\mathrm{X}$ & & MBS 113 \\
\hline
\end{tabular}




\begin{tabular}{|c|c|c|c|c|c|c|c|c|c|}
\hline Família & Espécie & Forma & IBI & IGR & PRA & FAS & GIG & PBA & $\begin{array}{l}\text { Coletor / } \\
\text { Amostra } \\
\end{array}$ \\
\hline & Habenaria cf. janeirensis Kranzl. & HER & $\mathrm{X}$ & & & & & & MBS 562 \\
\hline & Habenaria paranaensis Barb.Rodr. & HER & $\mathrm{X}$ & & & & & & AYM 191 \\
\hline & Habenaria parviflora Lindl. & HER & $\mathrm{X}$ & & & & $X$ & & AYM 190 \\
\hline & Oncidium donianum Batem. ex Baxter & HER & $\mathrm{X}$ & & & & & & MBS 125 \\
\hline & Oncidium sp. 1 & HER & & & & $\mathrm{X}$ & & & $\mathrm{AYM} \mathrm{s} / \mathrm{n}^{\circ}$ \\
\hline & Oncidium sp. 2 & HER & & & & & $\mathrm{X}$ & & $\mathrm{AYM} \mathrm{s} / \mathrm{n}^{\circ}$ \\
\hline & Prescottia stachyodes (Sw.) Lindl. & HER & & $\mathrm{X}$ & & & & & MBS 579 \\
\hline & Rodriguesia sp. & HER & & & & & $X$ & & AYM 207 \\
\hline & Sophronitis coccinea (Lind1.) Reichb. & EPI & $\mathrm{X}$ & $\mathrm{X}$ & $\mathrm{X}$ & & & $\mathrm{X}$ & MBS 114 \\
\hline & Zygopetalum mackayi Hook. & HER & $\mathrm{X}$ & & & & $\mathrm{X}$ & & MBS 559 \\
\hline \multirow[t]{3}{*}{ Orobanchaceae } & Esterhazya splendida J.C. Mikan & HER & $\mathrm{X}$ & $\mathrm{X}$ & & $X$ & & $\mathrm{X}$ & MBS 126 \\
\hline & Velloziella westermannii Dusén & $\mathrm{ARB}$ & $\mathrm{X}$ & & & $\mathrm{X}$ & & & MBS 637 \\
\hline & Orobanchaceae indeterminada & HER & $\mathrm{X}$ & & & & & & MBS 749 \\
\hline Oxalidaceae & Oxalis cf. rupestris A.St.-Hil. & HER & $\mathrm{X}$ & & & & & & AYM 192 \\
\hline Pentaphyllacaceae & Ternstroemia brasiliensis Camb. & ARV & $\mathrm{X}$ & $\mathrm{X}$ & $\mathrm{X}$ & & $\mathrm{X}$ & & $\mathrm{AYM} \mathrm{s} / \mathrm{n}^{\circ}$ \\
\hline Plantaginaceae & Plantago australis Lam. & HER & $\mathrm{X}$ & & & & & & MBS 567 \\
\hline \multirow[t]{30}{*}{ Poaceae } & Andropogon macrothrix Trinius & HER & $\mathrm{X}$ & & & & & & AYM 155 \\
\hline & Aulonemia fimbriatifolia L.G. Clark & HER & $\mathrm{X}$ & & & & & & MBS 456 \\
\hline & Calamagrostis longearistata (Wedd.) Hack. ex Sodiro & HER & $\mathrm{X}$ & & & & & & AYM s $/ n^{\circ}$ \\
\hline & Chusquea anelytroides Rupr. ex Doell & HER & $\mathrm{X}$ & $\mathrm{X}$ & & & & & AYM 198 \\
\hline & Chusquea mimosa McClure \& L.B. Sm. & HER & $\mathrm{X}$ & $\mathrm{X}$ & $\mathrm{X}$ & $\mathrm{X}$ & & $\mathrm{X}$ & AYM 118 \\
\hline & Chusquea pinifolia (Nees) Nees & HER & $\mathrm{X}$ & $\mathrm{X}$ & $\mathrm{X}$ & $\mathrm{X}$ & & & MBS 77 \\
\hline & $\begin{array}{l}\text { Chusquea sp. } \\
\text { Cortaderia selloana (Schult. \& Schult. f.) Asch. \& } \\
\text { Graebn. }\end{array}$ & HER & $\mathrm{X}$ & & & & $\mathrm{X}$ & & $\begin{array}{l}\text { AYM s } / n^{\circ} \\
\text { AYM s/no }\end{array}$ \\
\hline & Danthonia secundiflora Presl & HER & $\mathrm{X}$ & & & & & $\mathrm{X}$ & AYM 115 \\
\hline & Danthonia sp. 1 & HER & & $\mathrm{X}$ & & & & & MBS 672 \\
\hline & Danthonia sp. 2 & HER & & & & & & $\mathrm{X}$ & MBS 480 \\
\hline & Danthonia sp. 3 & HER & $\mathrm{X}$ & & & & & & MBS 676 \\
\hline & Deschampsia sp. & HER & $\mathrm{X}$ & & & & & & AYM 153 \\
\hline & Eriochrysis holcoides (Nees) Kuhlmann & HER & $\mathrm{X}$ & & & & & & MBS 525 \\
\hline & Panicum sabulorum L. var. sabulorum & HER & $\mathrm{X}$ & $\mathrm{X}$ & & $\mathrm{X}$ & & & MBS 524 \\
\hline & Panicum sp. 1 & HER & & & $\mathrm{X}$ & & & & MBS 487 \\
\hline & Panicum sp. 2 & HER & $\mathrm{X}$ & & & & & & MBS 481 \\
\hline & Paspalum polyphyllum Nees & HER & $\mathrm{X}$ & & & & $\mathrm{X}$ & & MBS 472 \\
\hline & Poidium calotheca (Trinius) Matthei & HER & $\mathrm{X}$ & & & & & & AYM 152 \\
\hline & Polypogon elongatus Kunth & HER & $\mathrm{X}$ & & & & & & AYM 154 \\
\hline & Stipa sellowiana Nees & HER & $\mathrm{X}$ & & & & & & MBS 522 \\
\hline & Poaceae indeterminada 1 & HER & & & & & $\mathrm{X}$ & & MBS 675 \\
\hline & Poaceae indeterminada 2 & HER & & & & $\mathrm{X}$ & & & MBS 478 \\
\hline & Poaceae indeterminada 3 & HER & $\mathrm{X}$ & & & & & & RTP 102 \\
\hline & Poaceae indeterminada 4 & HER & $\mathrm{X}$ & & & & & & RTP 105 \\
\hline & Poaceae indeterminada 5 & HER & & & & & & $\mathrm{X}$ & MBS 477 \\
\hline & Poaceae indeterminada 6 & HER & $\mathrm{X}$ & & & & & & MBS 483 \\
\hline & Poaceae indeterminada 7 & HER & $\mathrm{X}$ & & & & & & AYM 19 \\
\hline & Poaceae indeterminada 8 & HER & $\mathrm{X}$ & & & & & & MBS 485 \\
\hline & Poaceae indeterminada 9 & HER & $\mathrm{X}$ & & & & & & AYM 299 \\
\hline & Poaceae indeterminada 10 & HER & & & $\mathrm{X}$ & & & & MBS 488 \\
\hline \multirow[t]{4}{*}{ Polygalaceae } & Polygala linoides Poir. & HER & $\mathrm{X}$ & & & & & & AYM 199 \\
\hline & Polygala subverticillata Chodat & HER & $\mathrm{X}$ & & & & & & MBS 569 \\
\hline & Polygala sp. 1 & HER & $\mathrm{X}$ & & & & & & MBS 610 \\
\hline & Polygala sp. 2 & HER & $\mathrm{X}$ & & $\mathrm{X}$ & & & & MBS 521 \\
\hline Polygonaceae & Coccoloba persicaria $\mathrm{L}$. & $\mathrm{ARB}$ & $\mathrm{X}$ & & $\mathrm{X}$ & $\mathrm{X}$ & & & AYM 200 \\
\hline
\end{tabular}

FLORESTA, Curitiba, PR, v. 38, n. 4, p. 625-640, out./dez. 2008. 


\begin{tabular}{|c|c|c|c|c|c|c|c|c|c|}
\hline Família & Espécie & Forma & IBI & IGR & PRA & FAS & GIG & PBA & $\begin{array}{l}\text { Coletor } \\
\text { Amostra }\end{array}$ \\
\hline \multirow[t]{2}{*}{ Proteaceae } & Euplassa aff. nebularis Rambo \& Sleumer & ARV & $\mathrm{X}$ & & & & & & MBS 571 \\
\hline & Roupala rhombifolia Mart. ex Meissn. & $\mathrm{ARB}$ & $\mathrm{X}$ & & $\mathrm{X}$ & & & & AYM 202 \\
\hline Rosaceae & Rubus rosifolius Stokes & HER & $\mathrm{X}$ & & & & & & AYM s $/ n^{\circ}$ \\
\hline \multirow[t]{6}{*}{ Rubiaceae } & Coccocypselum condalia Pers. & HER & $\mathrm{X}$ & $\mathrm{X}$ & & $\mathrm{X}$ & $\mathrm{X}$ & $\mathrm{X}$ & AYM 127 \\
\hline & Coccocypselum $\mathrm{sp}$ & HER & $\mathrm{X}$ & $\mathrm{X}$ & $\mathrm{X}$ & $\mathrm{X}$ & & $\mathrm{X}$ & MBS 294 \\
\hline & Galianthe gertii E.L. Cabral & HER & $\mathrm{X}$ & & & $\mathrm{X}$ & & $\mathrm{X}$ & AYM 260 \\
\hline & $\begin{array}{l}\text { Galium hypocarpium (L.) Endl. ex Griseb. } \\
\text { ssp. indecorum (Cham. \& Scl.) Dempster }\end{array}$ & HER & $\mathrm{X}$ & $\mathrm{X}$ & $\mathrm{X}$ & $\mathrm{X}$ & & & MBS 67 \\
\hline & Galium sellowianum (Cham.) Walp. & HER & $\mathrm{X}$ & & & & & & MBS 576 \\
\hline & Galium sp. & HER & $\mathrm{X}$ & & & & & & MBS 506 \\
\hline Salicaceae & Xylosma pseudosalzmanii Sleumer & ARV & $\mathrm{X}$ & & & & & & AYM 180 \\
\hline Sapindaceae & Matayba cf. juglandifolia (Cambess.) Radlk. & ARV & & & & & $\mathrm{X}$ & & AYM 229 \\
\hline Smilacaceae & Smilax campestris Griseb. & TRE & $\mathrm{X}$ & $\mathrm{X}$ & $\mathrm{X}$ & $\mathrm{X}$ & $\mathrm{X}$ & $\mathrm{X}$ & AYM 128 \\
\hline Solanaceae & Brunfelsia cf. pilosa Plowman & $\mathrm{ARB}$ & $\mathrm{X}$ & & & & & & MBS 599 \\
\hline \multirow[t]{2}{*}{ Symplocaceae } & Symplocos sp. 1 & ARV & $\mathrm{X}$ & $\mathrm{X}$ & $\mathrm{X}$ & $\mathrm{X}$ & $\mathrm{X}$ & & AYM 86 \\
\hline & Symplocos sp. 2 & ARV & & $\mathrm{X}$ & & $\mathrm{X}$ & $\mathrm{X}$ & & AYM 231 \\
\hline Theaceae & Gordonia fruticosa (Schrad.) Her. Keng & ARV & $\mathrm{X}$ & $\mathrm{X}$ & $\mathrm{X}$ & & $\mathrm{X}$ & & AYM s $/ n^{\circ}$ \\
\hline Valerianaceae & Valeriana ulei Graebn. & HER & $\mathrm{X}$ & $\mathrm{X}$ & $\mathrm{X}$ & $\mathrm{X}$ & & $\mathrm{X}$ & MBS 73 \\
\hline Violaceae & Viola cerasifolia St.-Hil. & HER & $\mathrm{X}$ & & $\mathrm{X}$ & & & & AYM 237 \\
\hline \multirow[t]{2}{*}{ Winteraceae } & Drimys angustifolia Miers & ARV & $\mathrm{X}$ & $\mathrm{X}$ & $\mathrm{X}$ & & & & AYM 236 \\
\hline & Drimys brasiliensis Miers & ARV & & & & & $\mathrm{X}$ & & MBS 606 \\
\hline \multirow[t]{3}{*}{ Xyridaceae } & Xyris caroliniana Walt. & HER & $\mathrm{X}$ & & $\mathrm{X}$ & & & $\mathrm{X}$ & MBS 602 \\
\hline & Xyris dissitifolia Kral \& Wand. & HER & $\mathrm{X}$ & $\mathrm{X}$ & $\mathrm{X}$ & $\mathrm{X}$ & & $\mathrm{x}$ & AYM 120 \\
\hline & Xyris stenophylla L.A. Nilsson & HER & $\mathrm{X}$ & $\mathrm{X}$ & $\mathrm{X}$ & $\mathrm{X}$ & & & AYM 121 \\
\hline \multicolumn{10}{|c|}{ DIVISÃO GIMNOSPERMAE } \\
\hline Pinaceae & Pinus sp. & ARV & $\mathrm{X}$ & & & $\mathrm{X}$ & & & AYM s $/ n^{\circ}$ \\
\hline Podocarpaceae & Podocarpus sellowii Klotzsch ex Endl. & ARV & $\mathrm{X}$ & $\mathrm{X}$ & & & $\mathrm{X}$ & $\mathrm{X}$ & MBS 231 \\
\hline \multicolumn{10}{|c|}{\begin{tabular}{|c|} 
DIVISÃO PTERIDOPHYTA \\
\end{tabular}} \\
\hline Blechnaceae & Blechnum imperiale H. Chr. & HER & $\mathrm{X}$ & $\mathrm{X}$ & $\mathrm{X}$ & $\mathrm{X}$ & $\mathrm{X}$ & $\mathrm{X}$ & AYM 331 \\
\hline Dennstaedtiaceae & Pteridium arachnoideum (Kaulf.) Maxon & HER & $\mathrm{X}$ & & & & & & MBS 732 \\
\hline Dryopteridaceae & Rumohra adiantiformis (G. Forst.) Ching & HER & $\mathrm{X}$ & $\mathrm{X}$ & & & $\mathrm{X}$ & & MBS 706 \\
\hline \multirow[t]{3}{*}{ Gleicheniaceae } & Dicranopteris nervosa (Kaulf.) Maxon & HER & $\mathrm{X}$ & $\mathrm{X}$ & & & $\mathrm{X}$ & & AYM 12 \\
\hline & Gleichenella pectinata (Willd.) Ching. & HER & $\mathrm{X}$ & $\mathrm{X}$ & $\mathrm{X}$ & $\mathrm{X}$ & $\mathrm{X}$ & $\mathrm{X}$ & AYM 364 \\
\hline & Sticherus longipes (Fée) Copel. & HER & $\mathrm{X}$ & $\mathrm{X}$ & $\mathrm{X}$ & $\mathrm{X}$ & $\mathrm{X}$ & $\mathrm{X}$ & MBS 388 \\
\hline \multirow[t]{6}{*}{ Lycopodiaceae } & Huperzia quadrifariata (Bory) Rothm. & HER & & & $\mathrm{X}$ & & & & AYM 348 \\
\hline & Lycopodiella camporum B. Øllg. \& P.G. Windisch & HER & & & & & $\mathrm{X}$ & & MBS 730 \\
\hline & Lycopodiella caroliniana (L.) Pichi-Sermolli & HER & $\mathrm{X}$ & & & & $\mathrm{X}$ & $\mathrm{X}$ & AYM 350 \\
\hline & Lycopodium clavatum $\mathrm{L}$. & HER & $\mathrm{X}$ & & & $\mathrm{X}$ & & & MBS 747 \\
\hline & Lycopodium thyoides Humb. \& Bonpl. ex Willd. & HER & $\mathrm{X}$ & & & & & $\mathrm{X}$ & AYM 347 \\
\hline & Lycopodium sp. & HER & & & $\mathrm{X}$ & & & & MBS 518 \\
\hline \multirow[t]{2}{*}{ Polypodiaceae } & Cochlidium punctatum (Raddi) L.E. Bishop & EPI & $\mathrm{X}$ & $\mathrm{X}$ & $\mathrm{X}$ & & & & AYM 332 \\
\hline & Polypodium hirsutissimum Raddi & HER & $\mathrm{X}$ & & & & & & MBS 736 \\
\hline \multirow[t]{2}{*}{ Pteridaceae } & Doryopteris crenulans (Fée) H. Christ & HER & $\mathrm{X}$ & & $\mathrm{X}$ & & $\mathrm{X}$ & & AYM 362 \\
\hline & Pteridaceae indeterminada & HER & & & & & $\mathrm{X}$ & & AYM 17 \\
\hline Selaginellaceae & Selaginella $\mathrm{sp}$. & HER & & & & & $\mathrm{X}$ & & MBS 735 \\
\hline Indeterminada & Pteridophyta indeterminada & HER & & & & & $\mathrm{X}$ & & AYM 11 \\
\hline
\end{tabular}

A tabela 3 apresenta os resultados de trabalhos sobre a composição florística em campos de altitude no Brasil. Observa-se que os valores de riqueza específica encontrados no presente trabalho são consoantes com os demais estudos sobre os campos de altitude no Brasil.

A família que apresentou maior riqueza específica foi Asteraceae, com 44 espécies $(15,7 \%$ do total), seguida por Poaceae $(31 ; 11 \%)$, Melastomataceae $(17 ; 6 \%)$, Cyperaceae $(16 ; 5,7 \%)$, Ericaceae $(14$; 
5\%), Myrtaceae $(13 ; 4,6 \%)$ e Orchidaceae $(12 ; 4 \%)$. As outras famílias apresentaram até 10 espécies. A grande riqueza específica de Asteraceae já havia sido reportada em estudos de outras áreas de campos de altitude na região Sudeste do Brasil (RIZZINI, 1954; MARTINELLI, 1996; SAFFORD, 1999a). Esses estudos também observaram uma alta riqueza de Poaceae, Cyperaceae, Melastomataceae, Ericaceae, Orchidaceae, Lamiaceae, Lycopodiaceae e Rubiaceae em montanhas da Serra da Mantiqueira e da Serra do Mar. No campo de altitude estudado por Caiafa; Silva (2005), na Serra do Brigadeiro, as famílias mais importantes foram Orchidaceae (14 espécies), Asteraceae (12), Melastomataceae (8) e Cyperaceae (7). Chama a atenção neste estudo a baixa riqueza de Poaceae, com apenas duas espécies, ainda que estas tenham apresentado grande importância na estrutura da comunidade.

Tabela 3. Riqueza florística em campos de altitude em diferentes estudos no Brasil.

Table 3. Floristic richness in high altitude gross lands from different studies in Brazil.

\begin{tabular}{lcccc}
\hline Área de estudo & Localização & Estado & $\begin{array}{c}\text { Número de } \\
\text { espécies }\end{array}$ & Fonte \\
\hline Planalto do Itatiaia & Serra da Mantiqueira & RJ/MG & 415 & Martinelli, 1996 \\
\hline Campo das Antas (na Serra dos Órgãos) & Serra do Mar & RJ & 347 & Martinelli, 1996 \\
\hline Pedra do Desengano & Serra do Mar & RJ & 275 & Martinelli, 1996 \\
\hline Morro da Cuca & Serra do Mar & RJ & 227 & Martinelli, 1996 \\
\hline Serra da Bocaina & Serra do Mar & SP/RJ & 215 & Martinelli, 1996 \\
\hline Pico do Frade & Serra do Mar & RJ & 124 & Martinelli, 1996 \\
\hline $\begin{array}{l}\text { Serra Totem Deitado (na Serra do } \\
\text { Brigadeiro) }\end{array}$ & Serra da Mantiqueira & MG & 81 & Caiafa e Silva, 2005 \\
\hline Serra do Ibitiraquire & Serra do Mar & PR & 224 & Presente trabalho \\
\hline Serra da Igreja & Serra do Mar & PR & 99 & Presente trabalho \\
\hline Serra da Prata & Serra do Mar & PR & 93 & Presente trabalho \\
\hline Serra da Farinha Seca & Serra do Mar & PR & 85 & Presente trabalho \\
\hline Serra da Pedra Branca & Serra do Mar & PR & 71 & Presente trabalho \\
\hline Serra Gigante & Serra do Mar & PR & 80 & Presente trabalho \\
\hline Serra do Mar - total das 6 áreas acima & Serra do Mar & PR & 280 & Presente trabalho \\
\hline
\end{tabular}

Semelhanças quanto às famílias mais ricas também foram observadas em estudos com os campos rupestres. Falkenberg (2003), na região dos Aparados da Serra Geral, divisa dos estados de Santa Catarina e Rio Grande do Sul, observou Asteraceae com maior riqueza, abrangendo 18,5\% das espécies. Asteraceae, Poaceae, Cyperaceae e Melastomataceae também foram descrita como as mais ricas para os campos rupestres em Minas Gerais, na Serra da Bocaina (CARVALHO, 1992), na Serra do Brigadeiro (CAIAFA, 2002) e no Morro do Chapéu (ANDRADE et al., 1986).

Os gêneros que apresentaram maior riqueza foram Baccharis (18 espécies), Rhynchospora (7), Mikania (6), Mimosa (6), Leandra (6) e Tibouchina (5).

Apenas 17 espécies (6\% do total) foram encontradas em todas as áreas estudadas. São elas: Baccharis cf. trimera e Dendrophorbium limosum (Asteraceae), Vriesea platynema var. variegata (Bromeliaceae), Clethra uleana (Clethraceae), Lagenocarpus triquetrus e Machaerina austrobrasiliensis (Cyperaceae), Gaylussacia brasiliensis var. brasiliensis (Ericaceae), Ocotea tristis (Lauraceae), Utricularia reniformis (Lentibulariaceae), Leandra cf. quinquedentata e Miconia lymanii (Melastomataceae), Gomidesia sellowiana e Siphoneugena reitzii (Myrtaceae), Smilax campestris (Smilacaceae), Blechnum imperiale (Blechnaceae), Gleichenella pectinata e Sticherus longipes (Gleicheniaceae).

Também foram encontradas espécies descritas recentemente, como Alstroemeria amabilis (ASSIS, 2003), Aulonemia fimbriatifolia (CLARK, 2004) e Myrsine altomontana (FREITAS; KINOSHITA, 2005), além de espécies com sua descrição ainda não publicada, como Symplocos sp. 1 e Symplocos sp. 2, e uma nova espécie de Lamiaceae (Herperozygis sp.; Élide dos Santos, comunicação pessoal). Essa situação reflete o pouco esforço de pesquisa realizado nos campos de altitude no Paraná e reforça a importância de ações para sua conservação. 


\section{Riqueza florística e análise comparativa das áreas de estudo}

A maior riqueza de espécies foi encontrada na Serra do Ibitiraquire (224 espécies), seguida pelas serras da Igreja (99), da Prata (93), da Farinha Seca (85), Gigante (80) e da Pedra Branca do Araraquara (71).

A maior extensão dos campos de altitude está na Serra do Ibitiraquire, onde ocorre também o maior gradiente altitudinal (Tabela 1). Já a Serra da Pedra Branca do Araraquara, onde observou-se a menor riqueza de espécies, é, dentre as áreas estudadas, a que apresenta a menor cobertura pela formação. $\mathrm{Na}$ Serra Gigante, onde se encontrou baixa riqueza específica, os campos de altitude também ocorrem em pequenas áreas, próximas aos cumes. Nessas duas últimas áreas, o gradiente altitudinal de ocorrência da formação também é bastante restrito. A inexistência de dados oficiais e precisos a respeito da área ocupada pelos campos de altitude nos locais estudados impede inferências seguras sobre a relação entre a riqueza de espécies e a área ocupada pela formação que, a partir dos presentes resultados, parece existir.

Outro fator a ser considerado é a variação do esforço amostral. Ainda que ele tenha sido proporcional à extensão dos campos de altitude, algumas áreas, como a Serra da Igreja e a do Ibitiraquire, por serem maiores, foram amostradas em diversas ocasiões, abrangendo diferentes estações do ano, enquanto a Serra da Pedra Branca do Araraquara foi visitada apenas uma vez, numa fase de campo de dois dias. Ainda que o pequeno campo de altitude desta área tenha sido completamente percorrido nos dois dias de amostragem, uma nova fase de campo em outra estação do ano poderia revelar novas espécies.

A Serra do Ibitiraquire apresentou o maior número de espécies exclusivas (95), seguida pelas serras Gigante (20), da Pedra Branca do Araraquara (8), da Prata e da Farinha Seca (5) e da Igreja (4). No total, 137 espécies (49\%) foram observadas em apenas uma das áreas de amostragem.

A similaridade florística entre as áreas é alta (Tabela 4), sendo sempre superior a 0,25. Os maiores valores foram observados entre as serras da Prata, da Igreja e da Farinha Seca. Essas áreas estão relativamente próximas e apresentam pequena diferença latitudinal (Figura 1). Além disso, os campos de altitude dessas serras ocorrem em altitudes semelhantes, o que pode, em parte, explicar a grande similaridade florística. Poder-se-ia esperar uma composição florística diferente na Serra da Prata, em função de seu isolamento na planície litorânea, sem contato com o primeiro planalto paranaense, e da maior proximidade do oceano Atlântico. No entanto, isso não foi observado, e análises mais aprofundadas serão realizadas para um melhor entendimento dos padrões de distribuição das espécies.

Tabela 4. Índice da similaridade de Sorensen, em diferentes áreas amostradas. Table 4. Sorensen`s index of similarity at different sampled areas.

\begin{tabular}{lccccc}
\hline Locais & $\begin{array}{c}\text { Serra da } \\
\text { Igreja }\end{array}$ & $\begin{array}{c}\text { Serra da } \\
\text { Prata }\end{array}$ & $\begin{array}{c}\text { Serra da } \\
\text { Farinha Seca }\end{array}$ & Serra Gigante & $\begin{array}{c}\text { Serra da PB } \\
\text { Araraquara }\end{array}$ \\
\hline S. do Ibitiraquire (224) & 0,5201 & 0,5047 & 0,4660 & 0,3553 & 0,3797 \\
S. da Igreja (99) & - & 0,7292 & 0,6413 & 0,4246 & 0,5412 \\
S. da Prata (93) & & - & 0,6292 & 0,3815 & 0,5610 \\
S. da Farinha Seca (85) & & & - & 0,3152 & 0,5256 \\
S. Gigante (80) & & & & - & 0,3733 \\
S. da PB Araraquara (71) & & & & & - \\
\hline
\end{tabular}

A Serra Gigante apresentou a composição florística mais diferenciada. Um dos motivos pode estar na maior distância que a separa das demais áreas estudadas. A área estudada mais próxima, a Serra do Ibitiraquire, está a $65 \mathrm{~km}$, e o trecho de campo de altitude mais próximo está na Serra da Virgem Maria, a $40 \mathrm{~km}$ de distância. A ocorrência dos campos em altitudes relativamente menores também pode ter contribuído para a ocorrência de espécies exclusivas, tais como Aspidosperma pyricollum, Matayba cf. juglandifolia e Hedyosmum brasiliense, típicas das florestas montanas, entre 600 e $1000 \mathrm{~m}$ de altitude.

Os baixos valores de similaridade da Serra do Ibitiraquire estão relacionados com a grande riqueza de espécies e o grande número de espécies exclusivas.

A similaridade florística apresentou uma associação negativa e moderada com a distância entre as áreas $(\mathrm{r}=-0,78$; Figura 3$)$. A análise de agrupamento, apresentada na figura 2, ilustra os resultados da análise de similaridade entre as áreas.

\section{Ameaças à conservação dos campos de altitude no Paraná}

Durante os trabalhos de campo, diversas ameaças aos campos de altitude foram observadas, e considerou-se fundamental seu registro e divulgação. 


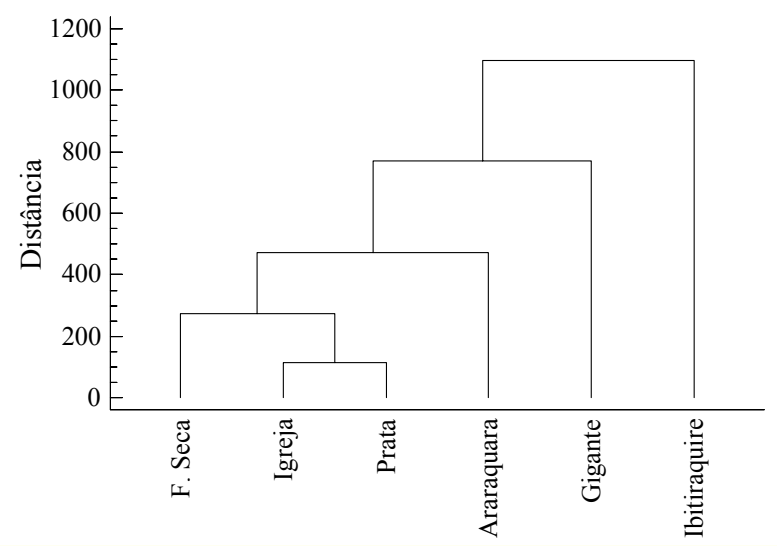

Figura 2. Dendrograma de agrupamento (Cluster) em função da presença ou ausência de espécies em cada área.

Figure 2. Cluster dendrogram in function of species presence and absence in each area.

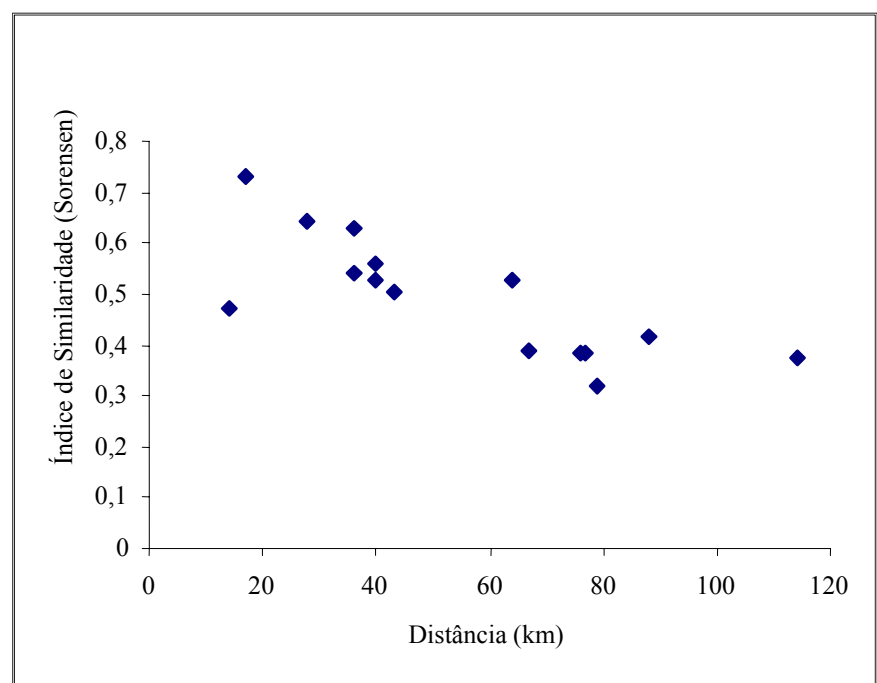

Figura 3. Relação entre similaridade florística e a distância entre as áreas amostradas através do coeficiente de correlação de Pearson $(r=-0,78)$.

Figure 3. Floristic similarity and distance association (Pearson; $r=-0,78$ ).

A contaminação biológica existe nos campos de altitude e a ocorrência de Pinus sp. é a mais preocupante. A contaminação na Serra do Ibitiraquire, que há poucos anos atingia apenas montanhas de menores altitudes, já atinge trechos mais elevados e em setores mais distantes das áreas antropizadas. Na Serra da Farinha Seca, um indivíduo foi detectado em uma montanha bastante isolada, pouquíssimo visitada por montanhistas, o que ilustra o potencial invasor da espécie. Foram encontradas outras espécies exóticas invasoras, como Lilium regale, Cortaderia selloana e Plantago australis, geralmente associadas a acampamentos. Também causa preocupação o registro da presença de animais domésticos junto a montanhistas, o que acarreta diversos impactos aos ecossistemas.

A conduta inadequada de pessoas em atividades recreativas também vem causando impactos significativos aos campos de altitude. Os mais comuns são o estabelecimento descontrolado de áreas de acampamento, abertura de trilhas e atalhos, uso inadequado do fogo e coleta de espécies vegetais para uso ornamental. Os impactos resultantes podem ser agravados com o estabelecimento de espécies exóticas invasoras nas áreas impactadas, que tendem a se propagar com maior vigor que a vegetação nativa em regeneração. 
As obras de infra-estrutura de telecomunicações ameaçam constantemente os ecossistemas em regiões montanhosas. Os problemas normalmente são agravados pelo fato de os executores das obras terem pouca ou nenhuma orientação quanto a técnicas de minimização de impactos. A instalação de uma antena de transmissão de ondas de rádio no Pico Caratuva, na Serra do Ibitiraquire, teve conseqüências desastrosas para os campos de altitude. Além da supressão da vegetação para a instalação dos equipamentos, foram abertas diversas clareiras desnecessárias. $\mathrm{O}$ transporte do material, feito inadequadamente por terra, gerou grandes impactos ao longo da trilha de acesso ao cume. Na mesma serra, em outra montanha, décadas após a construção de uma estrutura de telecomunicação, as conseqüências da falta de compromisso com a conservação do local ainda estão presentes. No local permanece grande quantidade de resíduos, como latões, vigas de aço e chapas metálicas, que foram literalmente descartadas em meio à vegetação. A preocupação com esse tipo de ameaça aumenta quando empresas de telefonia, visando atender metas de abrangência dos seus sinais, buscam as porções elevadas das serras para alocar suas antenas. Dessa forma, os restritos campos de altitude estarão permanentemente sob pressão.

A preocupação com a caça é uma constante em qualquer região da Serra do Mar. Na Serra da Igreja, onde foram registrados diversos vestígios de anta (Tapirus terrestris L.), também foram encontrados vários vestígios de caçadores (munições, cevas, etc.), inclusive nos campos de altitude. Além dos impactos diretos da caça, podem ocorrer impactos indiretos, como uso de fogueiras e presença de cachorros.

\section{CONCLUSÕES}

O presente trabalho apresenta a maior lista florística dos campos de altitude na Serra do Mar no Paraná, até o momento. Dessa forma, considera-se que o objetivo foi alcançado. No entanto, conforme comentado, não foram incorporados resultados de outros estudos e espécies registradas nos herbários que não foram detectadas neste trabalho. A inclusão dessas espécies elevaria, provavelmente de forma significativa, os valores de riqueza florística para a formação.

Asteraceae foi a família mais rica em todas as áreas estudadas. Também foram bastante expressivas Poaceae, Melastomataceae, Cyperaceae, Ericaceae, Myrtaceae e Orchidaceae. Os gêneros mais ricos foram Baccharis, Rhynchospora, Mikania, Mimosa, Leandra e Tibouchina. Esses resultados concordam com outros estudos em regiões campestres de altitude no Brasil. No entanto, notaram-se diferenças importantes quanto à ausência de espécies-chave reportadas em campos de altitude na região Sudeste do Brasil, havendo a necessidade de estudos comparativos mais aprofundados para um entendimento unificado da formação em toda sua área de ocorrência.

A Serra do Ibitiraquire apresentou a maior riqueza específica dos campos de altitude na Serra do Mar paranaense, devido provavelmente à maior área de ocorrência e ao maior gradiente altitudinal. Os campos de altitude nas serras da Prata, da Igreja e da Farinha Seca apresentaram composição florística bastante similar, provavelmente como conseqüência da proximidade geográfica e de semelhanças quanto à altitude de ocorrência. Os campos na Serra Gigante apresentaram a florística mais diferenciada, provavelmente pelo seu isolamento e baixa altitude.

Foram detectadas diversas ameaças à conservação dos campos de altitude no Paraná, que podem agir sinergicamente, ampliando os impactos sobre sua biodiversidade. Esses problemas são de difícil resolução, mas considerando que todas as áreas onde tais ameaças foram observadas são protegidas por unidades de conservação, ampliam-se as possibilidades de ações de proteção. Mesmo no caso das unidades de conservação ainda não implementadas, pode-se buscar o cumprimento da legislação ambiental básica, o que já evitaria grande parte dos impactos observados. No entanto, apenas a efetiva implementação das unidades de conservação, com recursos humanos bem dimensionados e capacitados, colocando em prática seus planos de manejo, asseguraria a proteção eficiente tanto para os campos de altitude quanto para os demais ecossistemas abrigados.

\section{AGRADECIMENTOS}

Agradecemos à Fundação O Boticário de Proteção à Natureza, pelo patrocínio do "Projeto Altomontana - A Floresta Ombrófila Densa Altomontana e os Refúgios Vegetacionais Altomontanos no Paraná”, à Sociedade Fritz Muller de Ciências Naturais, pelo apoio ao projeto, e ao PIBIC/CNPq, pela concessão de uma bolsa de iniciação científica. Agradecemos também às contribuições de Ruddy 
Proença, Samuel Arruda, Sandro M. Silva, Gustavo Gatti, Gilberto Tiepolo, Carlos V. Roderjan, Daniel Falkenberg, Gustavo Martinelli, Osmar S. Ribas, Gert G. Hatschbach, Juarez Cordeiro, Edimilson Costa, Marcos Sobral, Renato Goldenberg, Paulo Labiak, Armando C. Cervi, Élide dos Santos, Olavo A. Guimarães, Marília Borgo, Carina Kozera, Rodrigo A. Kersten e Marcelo Brotto.

\section{REFERÊNCIAS}

AB'SÁBER, A. N. Summit surfaces in Brazil. Revista Brasileira de Geociências, São Paulo, v. 30, n. 3, p. 515-516, 2000.

ALMEIDA, F. F. M. de; CARNEIRO, C. D. Origem e evolução da Serra do Mar. Revista Brasileira de Geociências, São Paulo, v. 28, n. 2, p. 135-150, 1997.

ANDRADE, P. M.; GONTIJO, T. A.; GRANDI, T. S. M. Composição florística e aspectos estruturais de uma área de campo rupestre do Morro do Chapéu, Nova Lima, MG. Revista Brasileira de Botânica, São Paulo, v. 9, n. 1, p. 13-21, 1986.

APG II 2003. An update of the Angiosperm Phylogeny Group classification for the orders and families of flowering plants: APG II. Botanical Journal of the Linnean Society, London, v. 141, p. 399-436, 2003.

ASSIS, M. C. Duas novas espécies de Alstroemeria L. (Alstroemeriaceae) para o Brasil. Acta Botânica Brasílica, Porto Alegre, v. 17, n. 2, p. 179-182, 2003.

BIGARella, J. J. A Serra do Mar e a porção oriental do estado do Paraná, um problema de segurança ambiental e nacional (contribuição à geografia, geologia e ecologia regional).. Curitiba: SEP; ADEA, 1978.

CAIAFA, A. N.; SILVA, A. F. da. Composição florística de um campo de altitude no Parque Estadual da Serra do Brigadeiro, Minas Gerais - Brasil. Rodriguésia, Rio de janeiro, v. 56, n. 87, p. 163-173, 2005.

CAIAFA, A. N. Composição florística e estrutura da vegetação sobre um afloramento rochoso no Parque Estadual da Serra do Brigadeiro, MG. Dissertação (Mestrado) - Universidade Federal de Viçosa, Viçosa, MG, 2002.

CARVALHO, D. A. Flora fanerogâmica de campos rupestres da Serra da Bocaina, Minas Gerais: caracterização e lista de espécies. Ciência e Prática, Lavras, v. 16, n. 1, p. 97-122, 1992.

CLARK, L. G. New species of Aulonemia and Chusquea (Poaceae: Bambusoideae: Bambuseae) from southeastern Brazil. Revista Brasileira de Botânica, São Paulo, v. 27, n. 1, p. 31-36, 2004.

FALKENBERG, D. B. Matinhas nebulares e vegetação rupícola dos Aparados da Serra Geral (SC/RS), sul do Brasil. Tese (Doutorado) - Instituto de Biociências, Universidade de Campinas, Campinas, 2003.

FERRI, M. G. Vegetação Brasileira. São Paulo: Editora da Universidade de São Paulo. 1980. 157 p.

FREITAS, M. F.; KINOSHITA, L. S. Novas espécies de Myrsine L. (Myrsinaceae) para o Brasil. Rodriguésia, Rio de Janeiro, v. 56, n. 87, p. 67-72, 2005.

IBGE (INSTITUTO BRASILEIRO DE GEOGRAFIA E ESTATÍSTICA). Manual técnico da vegetação brasileira. Rio de Janeiro, 1992. (Manuais Técnicos de Geociências, n.1).

LEITE, P. F. As diferentes unidades fitoecológicas da Região Sul do Brasil: proposta de classificação. Dissertação (Mestrado em Engenharia Florestal) - Setor de Ciências Agrárias, Universidade Federal do Paraná, Curitiba, 1994.

MAACK, R. 2002. Geografia física do Estado do Paraná. Curitiba. Imprensa Oficial. 440 p.

MARTINELLI, G. Campos de altitude. Rio de Janeiro: Index, 1996.

OLIVEIRA-FILHO, A. T.; FLUMINHAN-FILHO, M. Ecologia da vegetação do Parque Florestal Quedas do Rio Bonito. Revista Cerne, Lavras, v. 5, n. 2, p. 51-64, 1999. 
PARANÁ. Secretaria de Estado do Meio Ambiente; Instituo Ambiental do Paraná. PRÓ-ATLÂNTICA - Programa de Proteção da Floresta Atlântica: Projeto Carta Geológica: Cartas: MI-2844-2 Ariri, MI2843-1 Represa do Capivari, MI-2843-3 Morretes, MI-2858-1 Mundo Novo e MI-2858-3 Pedra Branca do Araraquara. Escala 1:50000. Curitiba, 2002.

POREMBSKI, S.; MARTINELLI, G.; OHELMÜLLER, R.; BARTHLOTT, W. Diversity and ecology of saxicolous vegetation mats on inselbergs in the Brazilian Atlantic rainforest. Diversity and Distributions, Oxford, v. 4, p. 107-119, 1998.

RIBEIRO, K. T.; MEDINA, B. M. O. 2002. Estrutura, dinâmica e biogeografia das ilhas de vegetação sobre rocha do planalto do Itatiaia - RJ. Boletim do Parque Nacional do Itatiaia - RJ, Rio de Janeiro, n. $10.83 \mathrm{p}$.

RIZZINI, C. T. 1979. Tratado de fitogeografia do Brasil. São Paulo: Hucitec, 1979. 374 p.

RIZZINI, C. T. Flora Organensis. Arquivos do Jardim Botânico do Rio de Janeiro, Rio de Janeiro, v. 13, p. 118-243, 1954 ..

RODERJAN, C. V. Caracterização da vegetação dos Refúgios Vegetacionais Altomontanos (campos de altitude) nas serras dos órgãos e do Capivari no Estado do Paraná. Curitiba: [s.n.], 1999. Relatório Técnico do CNPq

RODERJAN, C. V.; GRODSKI, L. Acompanhamento meteorológico em um ambiente de Floresta Ombrófila Densa Altomontana no morro Anhangava, Mun. De Quatro Barras - PR, no ano de 1993. Cadernos da Biodiversidade, Maringá, v. 2, n. 1, p. 27-34, 1999.

RODERJAN, C. V., GALVÃO, F., KUNIYOSHI, Y. S.; HATSCHBACH, G. G. As unidades fitogeográficas do estado do Paraná. Ciência e Ambiente, Santa Maria, RS, v. 24, p. 75-92, 2002.

SAFFORD, H. D. Brazilian Paramos I. An introduction to the physical environment end vegetation of the campos de altitude. Journal of Biogeography, Oxford, v. 26, p. 693-712, 1999a.

SAFFORD, H. D. Brazilian Páramos II. Macro and mesoclimate of the campos de altitude and affinities with high mountain climates of tropical Andes and Costa Rica. Journal of Biogeography, Oxford, v. 26, p. 713-737, 1999b.

SAFFORD, H. D.; MARTINELLI, G. Southeast Brazil. In.: POREMBSKI, S.; BARTHLOTT, E. W. (Eds.). Inselbergs. Berlin: Springer-Verlag, 2000. (Ecological Studies, v. 146).

TRAMUJAS, A. P. A vegetação dos campos de altitude na região do Ibitiraquire - Serra do Mar Municípios de Antonina, Morretes e Campina Grande do Sul, Paraná. Dissertação (Mestrado em Engenharia Florestal) - Setor de Ciências Agrárias, Universidade Federal do Paraná, Curitiba, 2000.

TRYON, R. M.; TRYON, A. F. Ferns and allied plants. New York: Springer Verlag. 1982. 896 p.

VELOSO, H. P.; RANGEL FILHO, A. L. R.; LIMA, J. C. A. Classificação da vegetação brasileira, adaptada a um sistema universal. Rio de Janeiro: IBGE, 1991.

WISNIEWSKI, C.; TEMPESTA, P. B.; RODRIGUES, V. M. R. Solos e vegetação em uma topossequência do morro Mãe Catira, alto da Serra da Graciosa, Morretes PR.. In: CONGRESSO BRASILEIRO DE CIÊNCIA DO SOLO, 30., 2005, Recife. Anais... Viçosa, MG: SBCS. 2005. 